\title{
Ruthenium-Catalyzed Heck-Type Alkenylation of Alkyl Bromides
}

José María Muñoz-Molina, ${ }^{*}$ Pedro J. Pérez*

Laboratorio de Catálisis Homogénea, Unidad Asociada al CSIC, CIQSO-Centro de Investigación en Química Sostenible and Departamento de Química, Universidad de Huelva, 21007 Huelva, Spain

$\begin{array}{lll}\text { I Effect of reaction parameters } & \text { S-2 }\end{array}$

II Reaction monitoring $\quad$ S-2

III Oxidation and reduction monitored by ${ }^{31} \mathrm{P}\left\{{ }^{1} \mathrm{H}\right\} \mathrm{NMR} \quad \mathrm{S}-3$

IV Products mixture of the alkenylation leading to $\mathbf{3 j} \quad$ S-6

$\begin{array}{lll}\text { V NMR spectra } & \text { S-7 }\end{array}$

$\begin{array}{lll}\text { VI X-ray crystallographic data for 3d } & \text { S-26 }\end{array}$

$\begin{array}{llr}\text { VII } & \text { References } & \text { S-27 }\end{array}$ 


\section{Effect of reaction parameters}<smiles>C=Cc1ccc(OC)cc1</smiles>

1a

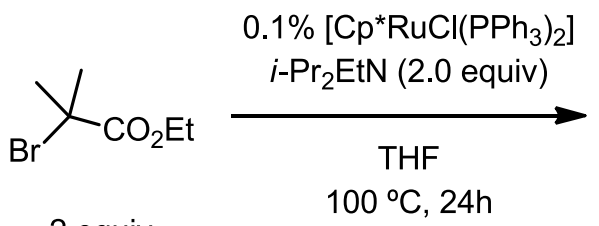

2a<smiles>CCOC(=O)C(C)(C)/C=C/c1ccc(OC)cc1</smiles>

3a

Under an atmosphere of $\mathrm{N}_{2}$, a glass ampoule equipped with a stir bar was charged in turn with the catalyst $(0.0002 \mathrm{mmol}, 0.1 \%)$ from a THF stock solution, the olefin $(0.2$ $\mathrm{mmol})$, the alkyl bromide $(0.4 \mathrm{mmol}), \mathrm{N}, \mathrm{N}$-diisopropylethylamine $(0.4 \mathrm{mmol})$, and dry THF to a total volume of $1 \mathrm{~mL}$. The ampoule was sealed with a Teflon screw cap, and then the reaction mixture was stirred at the desired temperature. After the reaction time, the ampoule was cooled down to room temperature and the volatiles were removed under reduced pressure. Diphenylmethane $(0.2 \mathrm{mmol}, 33.4 \mu \mathrm{L})$ was added, and the reaction was anlyzed by ${ }^{1} \mathrm{H} \mathrm{NMR}\left(\mathrm{CDCl}_{3}\right)$.

\section{Reaction monitoring}

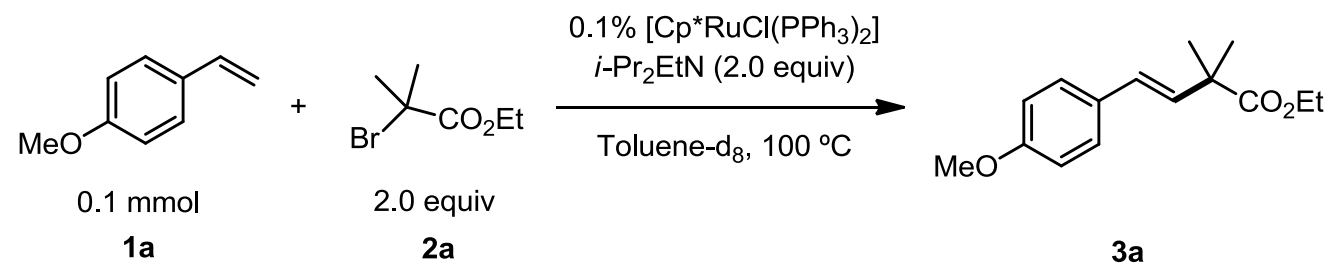

In a glovebox, the complex $\left[\mathrm{Cp} * \mathrm{RuCl}\left(\mathrm{PPh}_{3}\right)_{2}\right](0.0001 \mathrm{mmol}, 0.1 \%)$ from a toluene- $\mathrm{d}_{8}$ stock solution, 1a $(0.1 \mathrm{mmol}), 2 \mathrm{a}(0.2 \mathrm{mmol})$, and $N, N$-diisopropylethylamine $(0.2$ $\mathrm{mmol}$ ), and Diphenylmethane (internal standard) were added to a vial via syringe. Then, the required amount of toluene- $d_{8}$ was added to reach a total volume of $1.0 \mathrm{~mL}$ $\left([1 \mathrm{a}]_{0}=0.1 \mathrm{M}\right)$. The mixture was transferred to an NMR pressure tube sealed with a Teflon screw cap and placed in an oil bath at 100 으. The early stage of the reaction was then monitored by ${ }^{1} \mathrm{H}$ NMR spectroscopy using a delay $D_{1}=10 \mathrm{~s}$. The graphical representation of the extrapolated formation of $3 a(m M)$ vs time $(\mathrm{s})$ is shown below. 


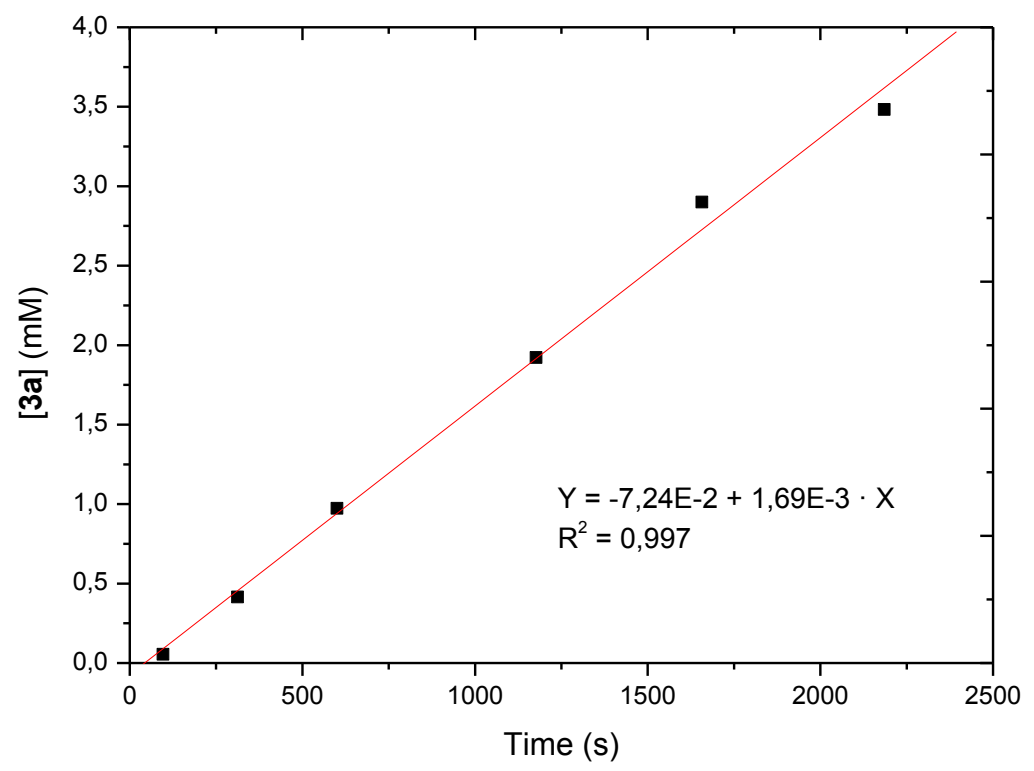

II. Oxidation and reduction monitored by ${ }^{31} \mathrm{P}\left\{{ }^{1} \mathrm{H}\right\}$ NMR

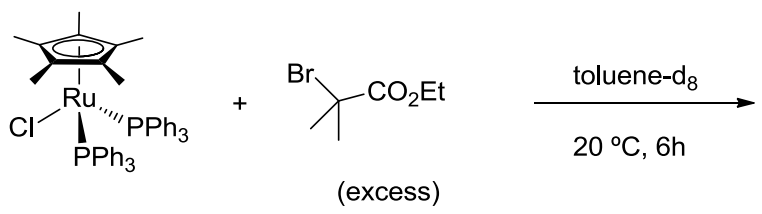

Ru(II) Complex

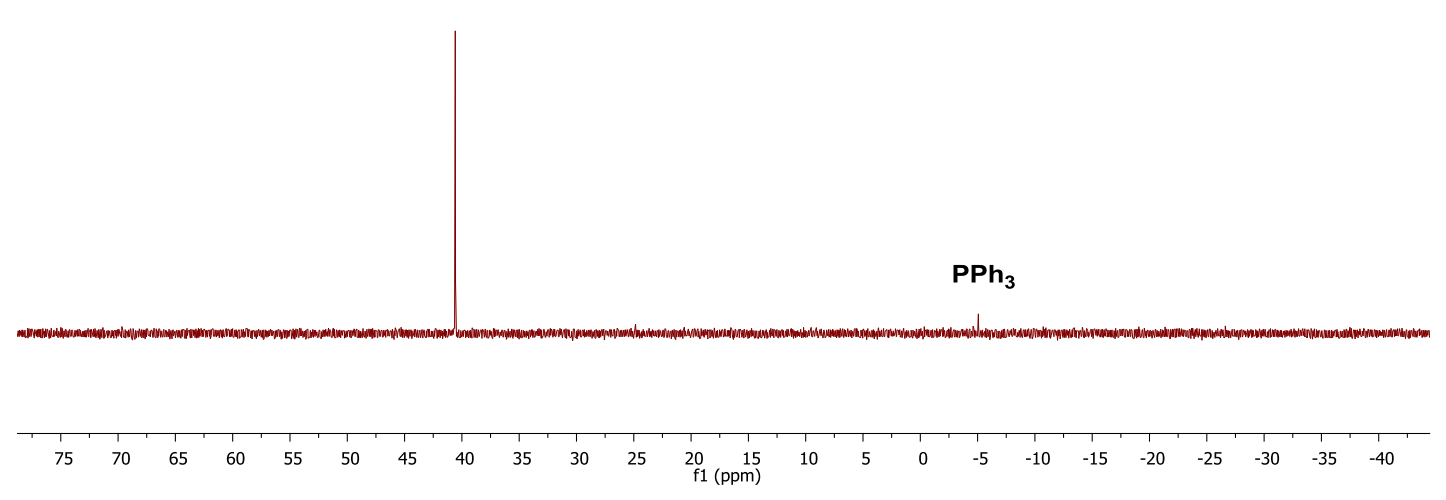




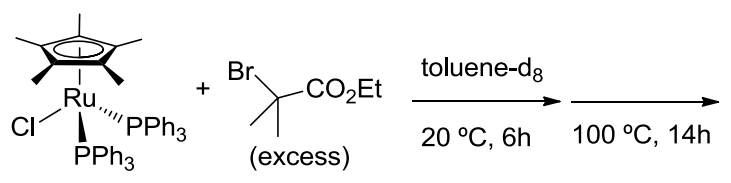

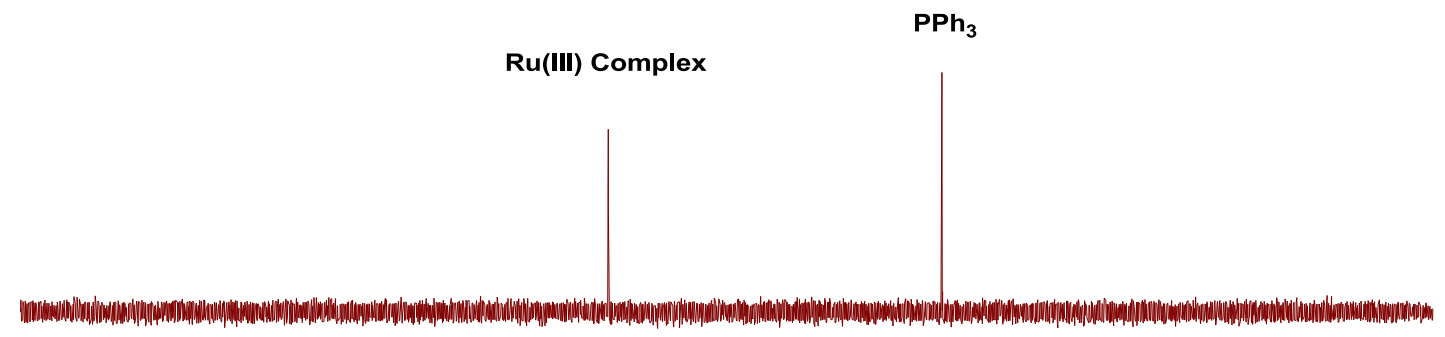

$\begin{array}{rlllllllllllllllllllllllll}75 & 70 & 65 & 60 & 55 & 50 & 45 & 40 & 35 & 30 & 25 & 20 & 15 & 10 & 5 & 0 & -5 & -10 & -15 & -20 & -25 & -30 & -35 & -40 & -45\end{array}$
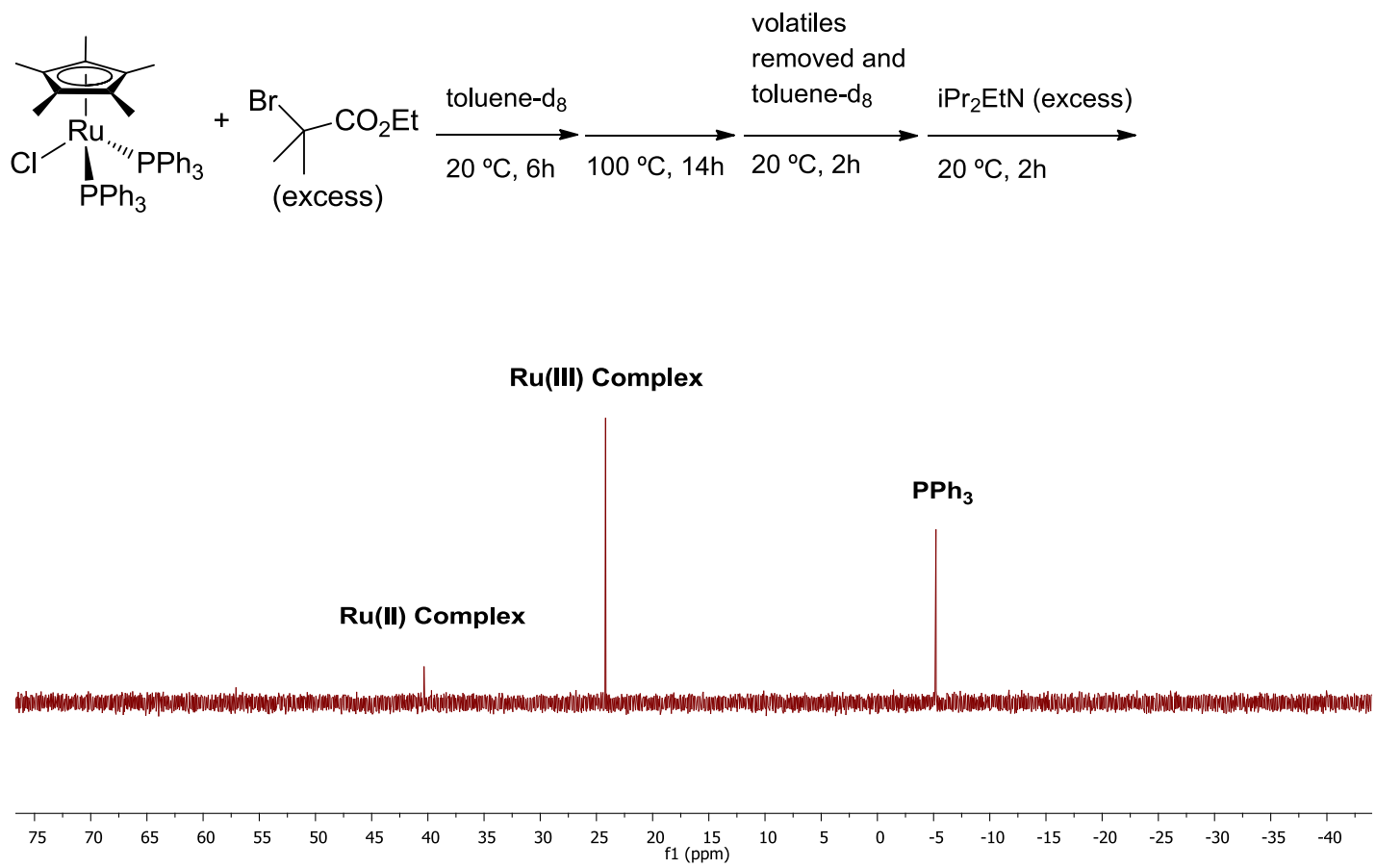


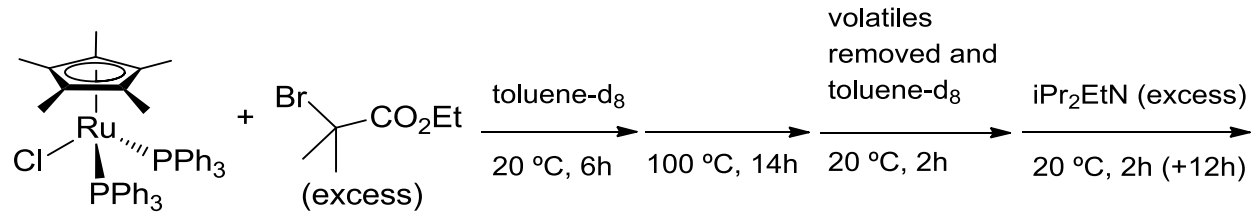

\section{Ru(III) Complex}
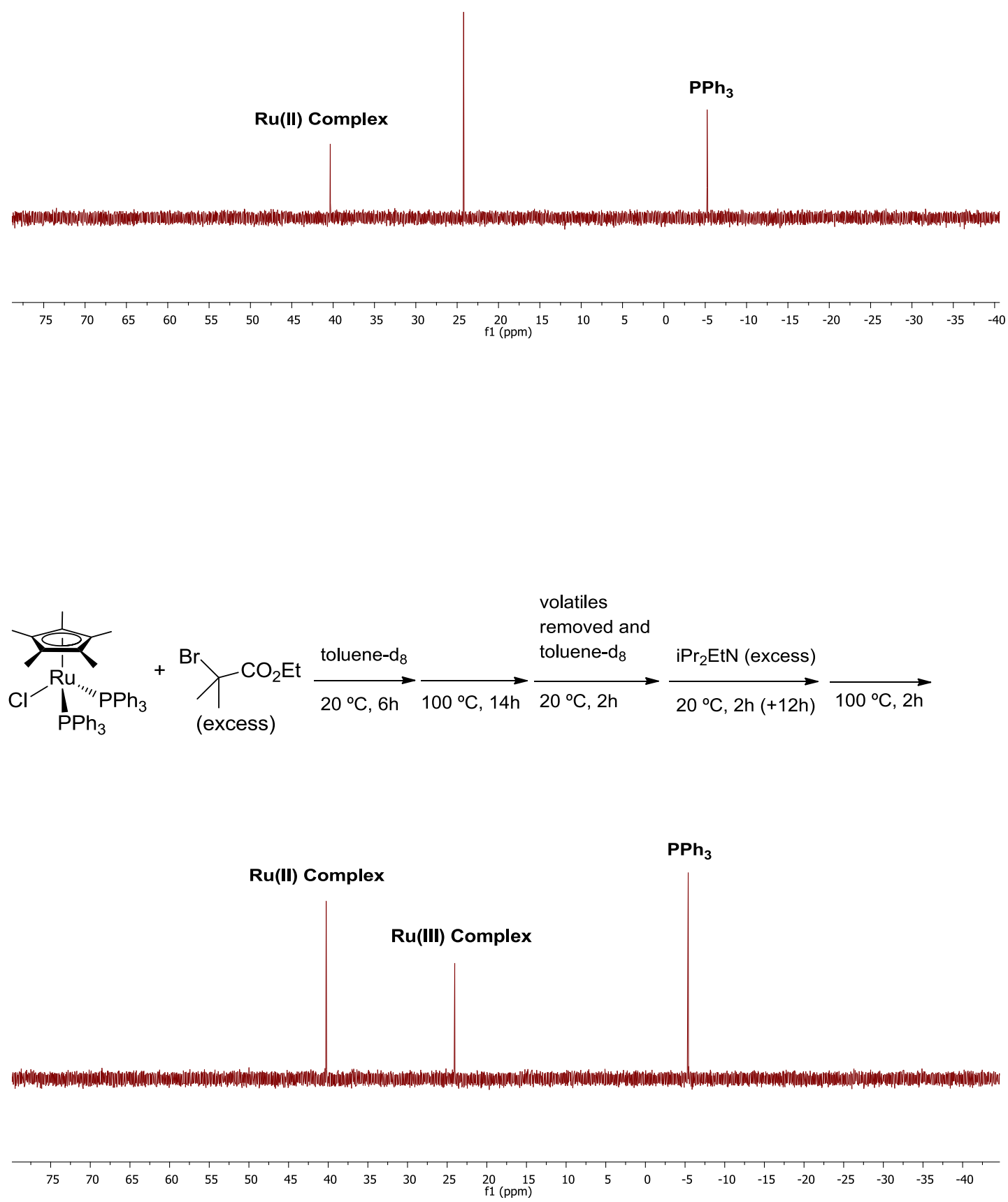


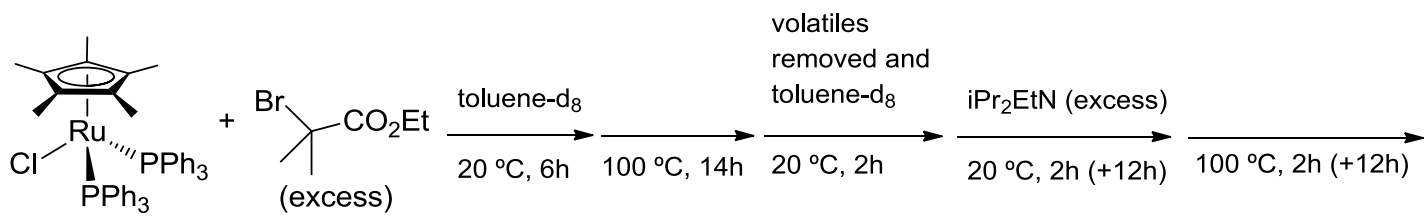

Ru(III) Complex

Ru(II) Complex

$\mathrm{PPh}_{3}$

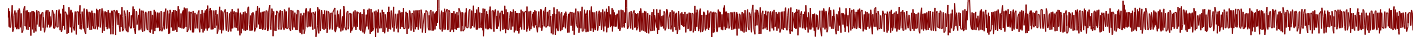

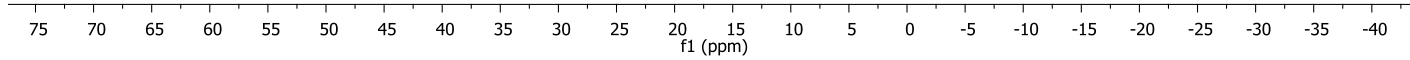

\section{Products mixture of the alkenylation leading to $\mathbf{3 j}$}
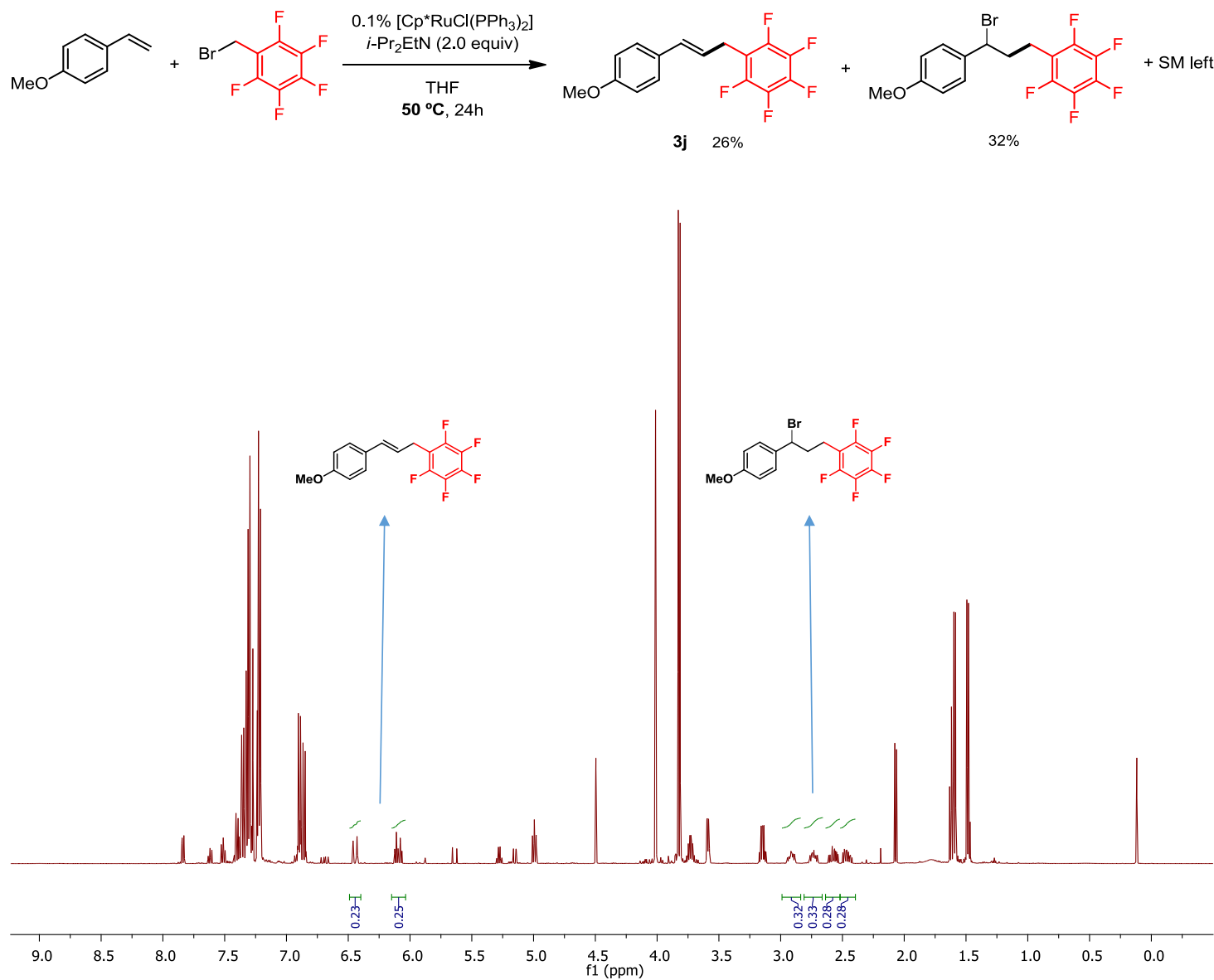


\section{NMR Spectra of Compounds}

3a, [1380341-70-7]

${ }^{1} \mathrm{H}$ NMR $\left(400 \mathrm{MHz}, \mathrm{CDCl}_{3}\right)$

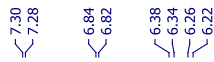

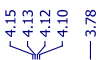

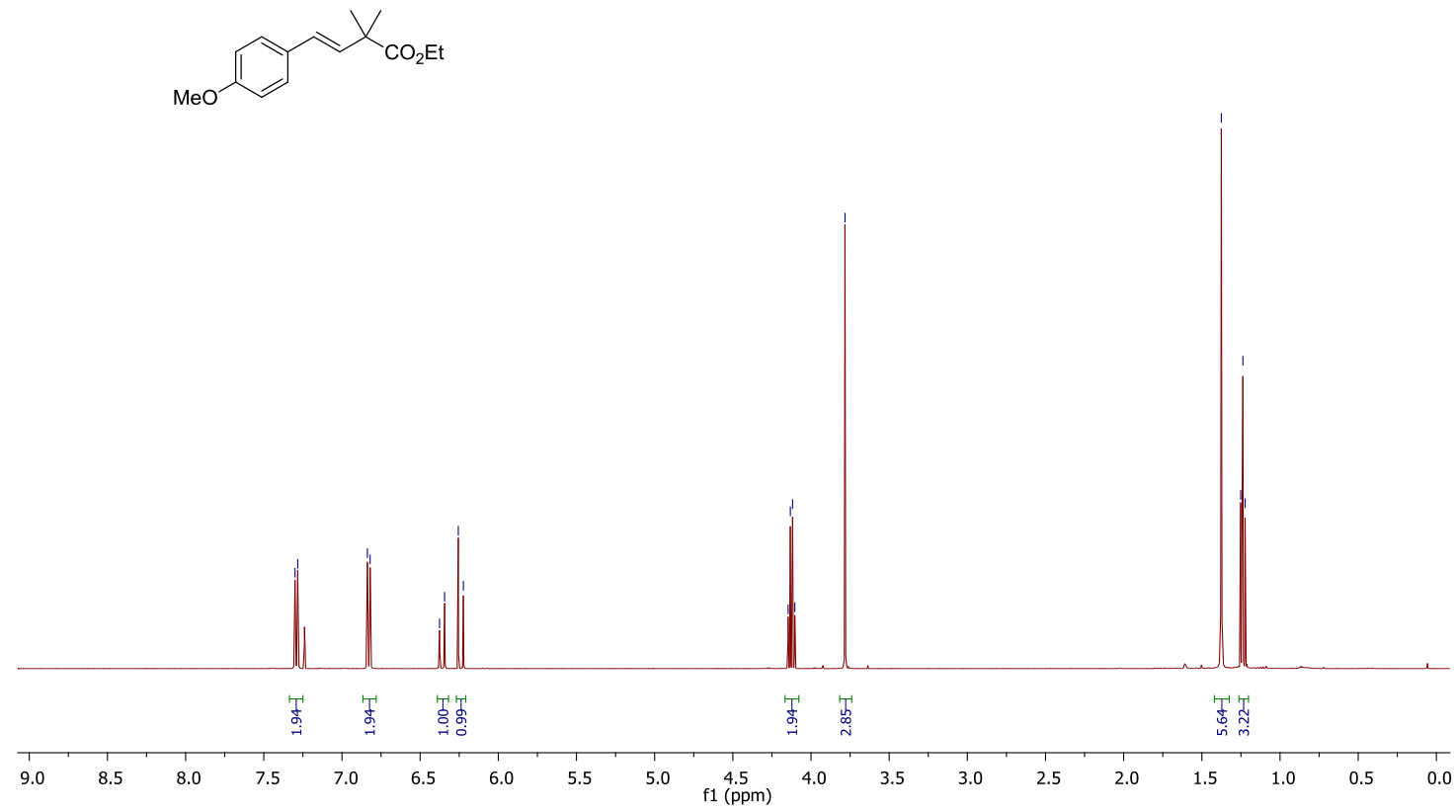

${ }^{13} \mathrm{C}\left\{{ }^{1} \mathrm{H}\right\}$ NMR $\left(126 \mathrm{MHz}, \mathrm{CDCl}_{3}\right)$

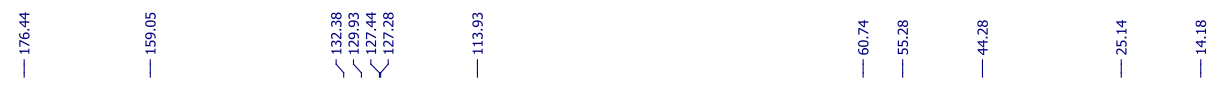

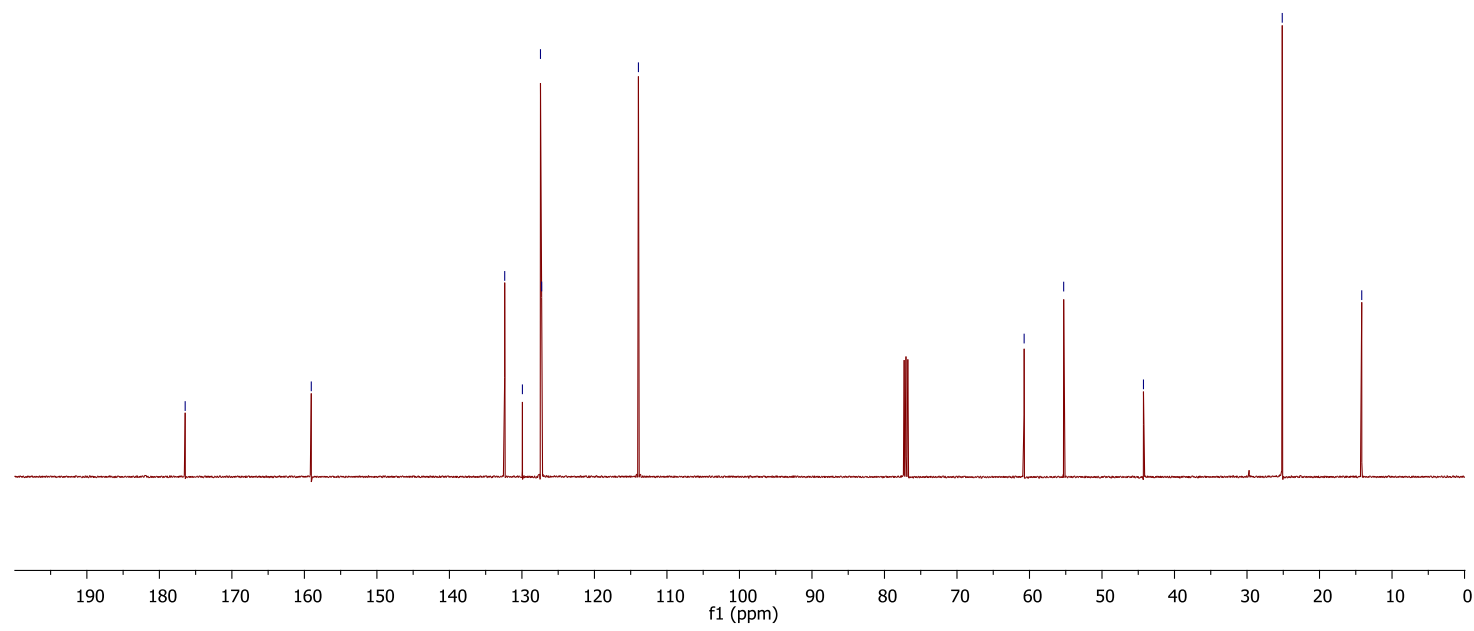


3b, [861391-88-0]

${ }^{1} \mathrm{H}$ NMR (400 MHz, $\mathrm{CDCl}_{3}$ )

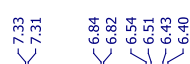

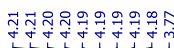

$\sqrt{1}$
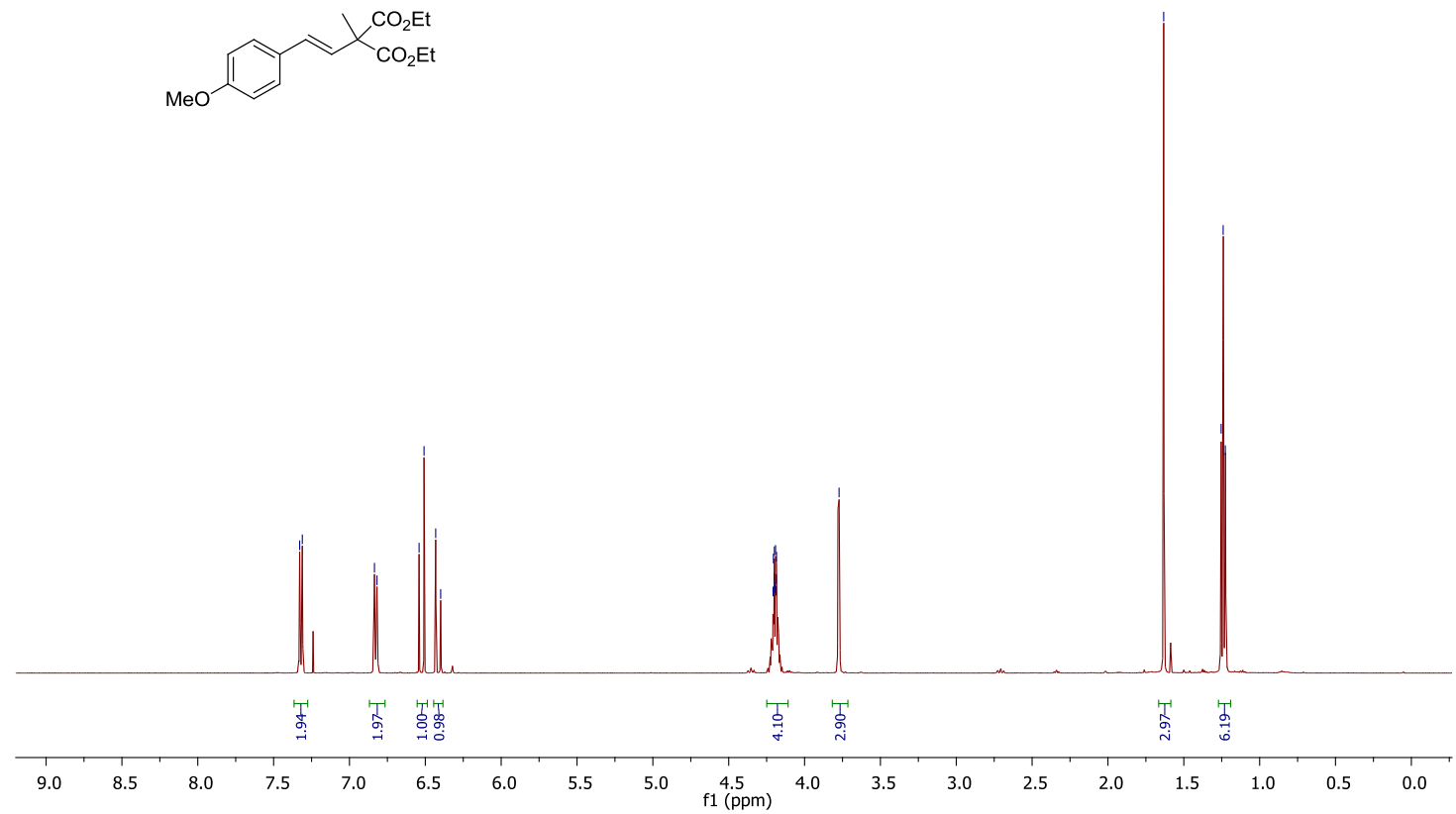

${ }^{13} \mathrm{C}\left\{{ }^{1} \mathrm{H}\right\}$ NMR $\left(126 \mathrm{MHz}, \mathrm{CDCl}_{3}\right)$

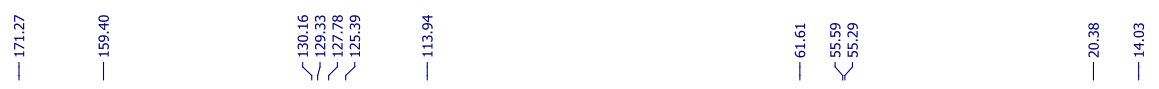

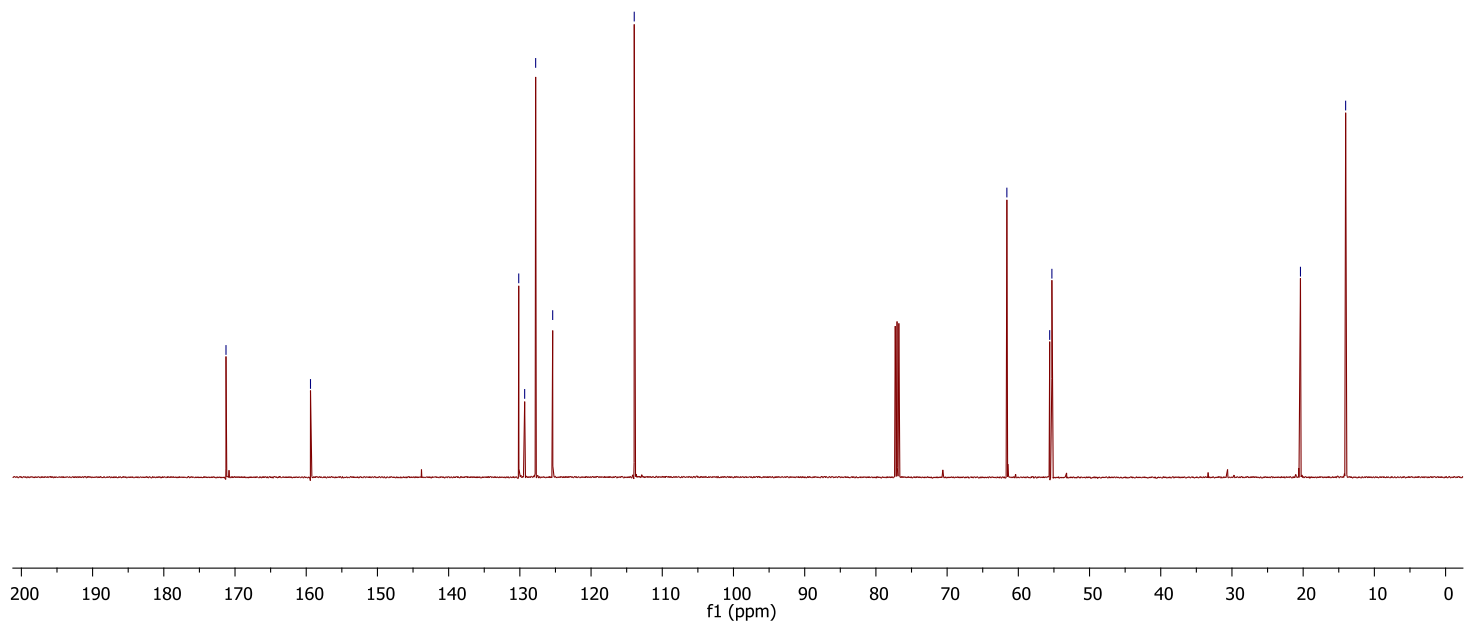


3c, [1473382-16-9]

${ }^{1} \mathrm{H}$ NMR (400 MHz, $\mathrm{CDCl}_{3}$ )

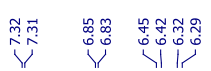

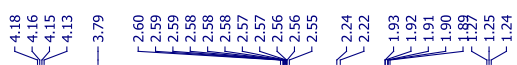

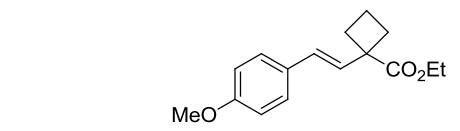

${ }^{13} \mathrm{C}\left\{{ }^{1} \mathrm{H}\right\} \mathrm{NMR}\left(126 \mathrm{MHz}, \mathrm{CDCl}_{3}\right)$

\begin{tabular}{|c|c|c|}
\hline & 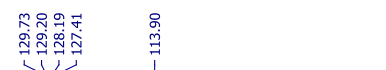 & \\
\hline
\end{tabular}

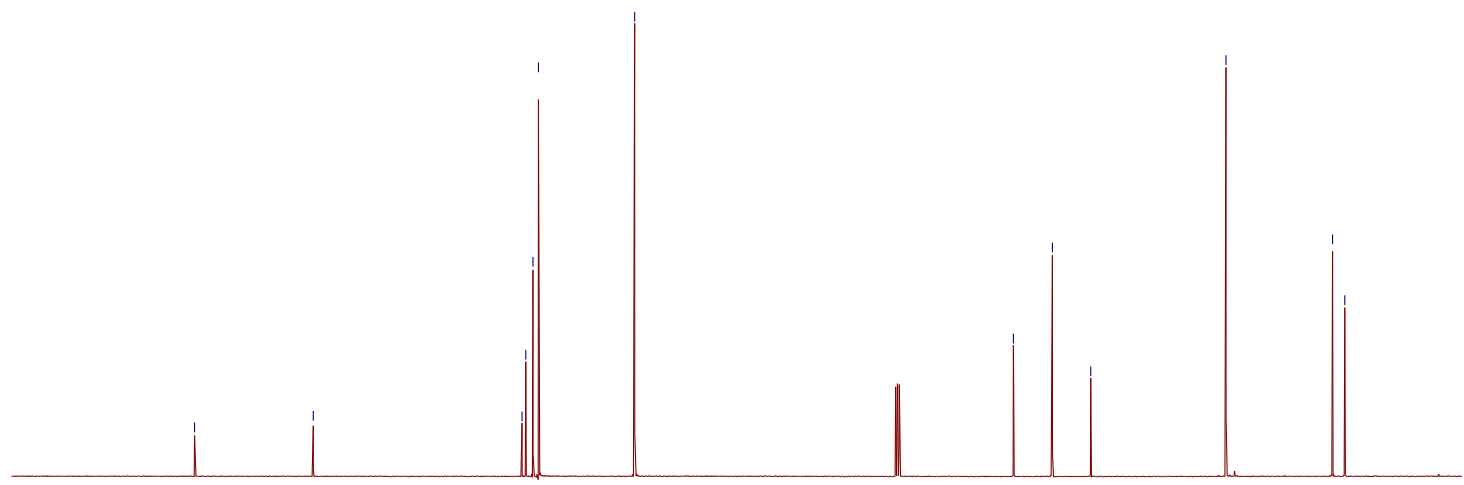

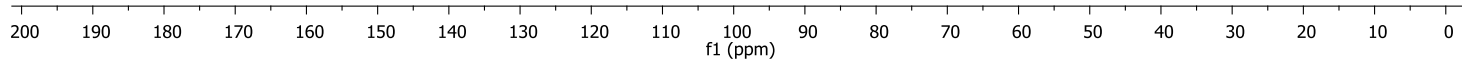


3d

${ }^{1} \mathrm{H}$ NMR (400 MHz, $\mathrm{CDCl}_{3}$ )

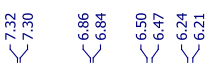

$\stackrel{\infty}{i}$

$\mathrm{M}_{\mathrm{M}}^{\mathrm{NH}_{2}}$
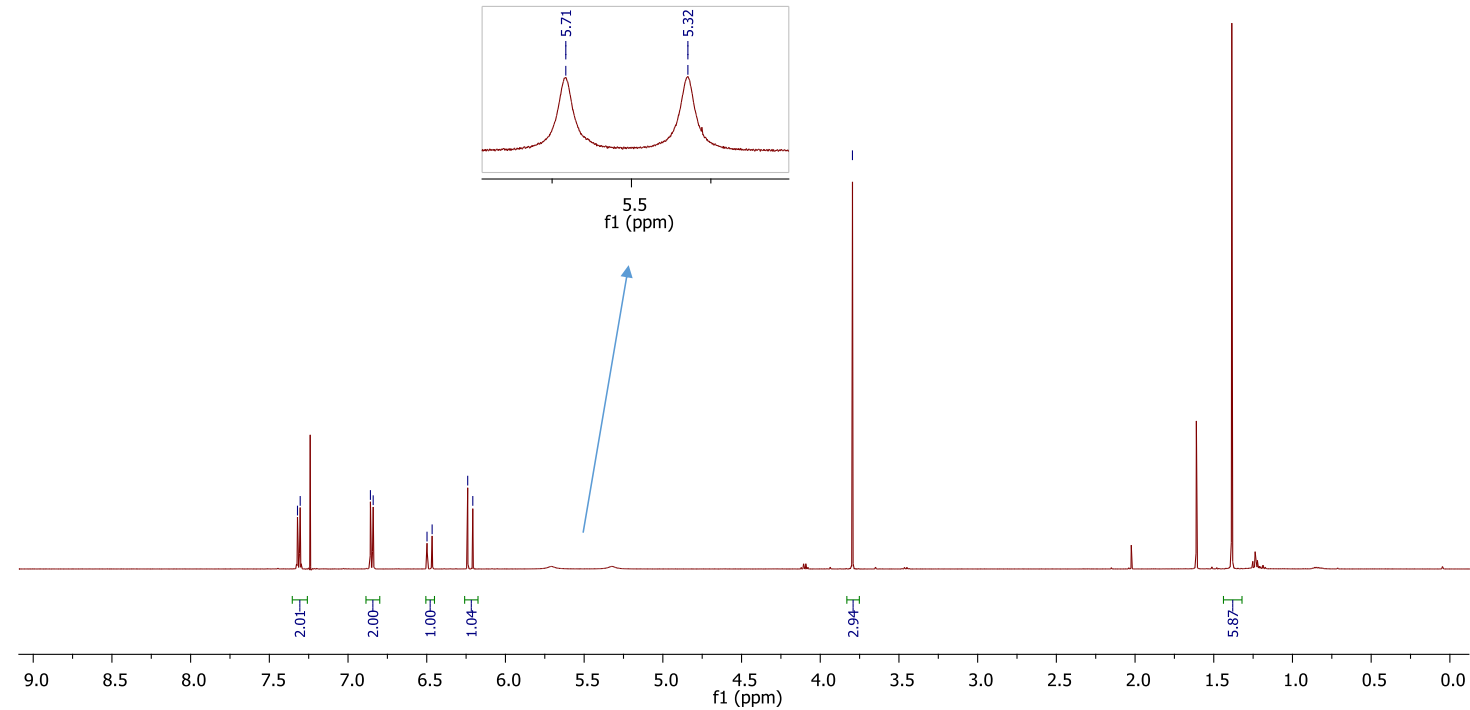

${ }^{13} \mathrm{C}\left\{{ }^{1} \mathrm{H}\right\}$ NMR $\left(126 \mathrm{MHz}, \mathrm{CDCl}_{3}\right)$

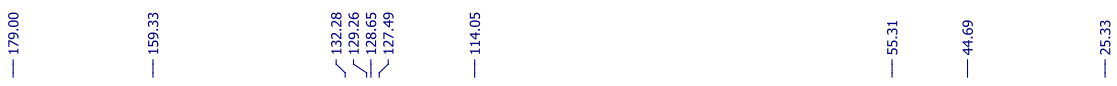

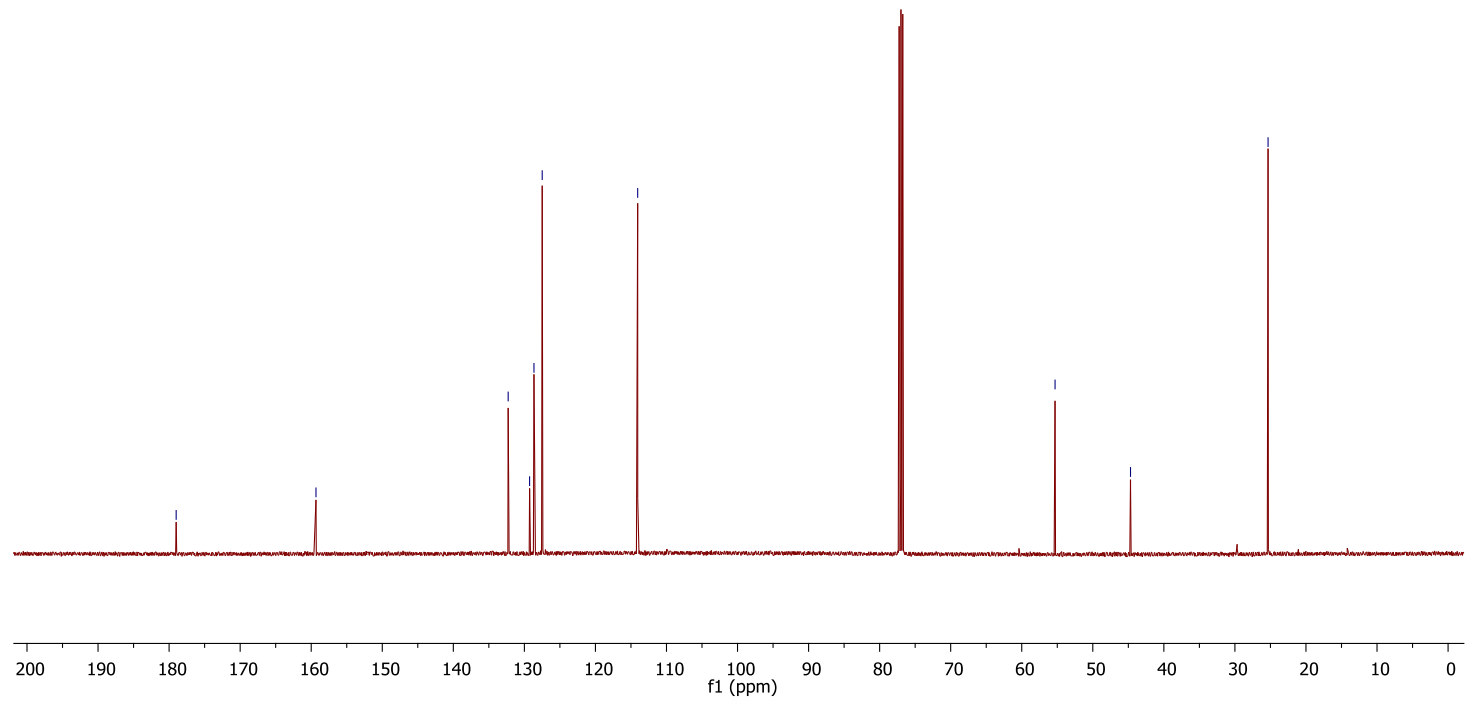


$3 e$

${ }^{1} \mathrm{H}$ NMR $\left(400 \mathrm{MHz}, \mathrm{CDCl}_{3}\right)$

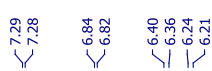

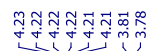

$\stackrel{\infty}{i} \stackrel{\substack{1 \\ i}}{i}$

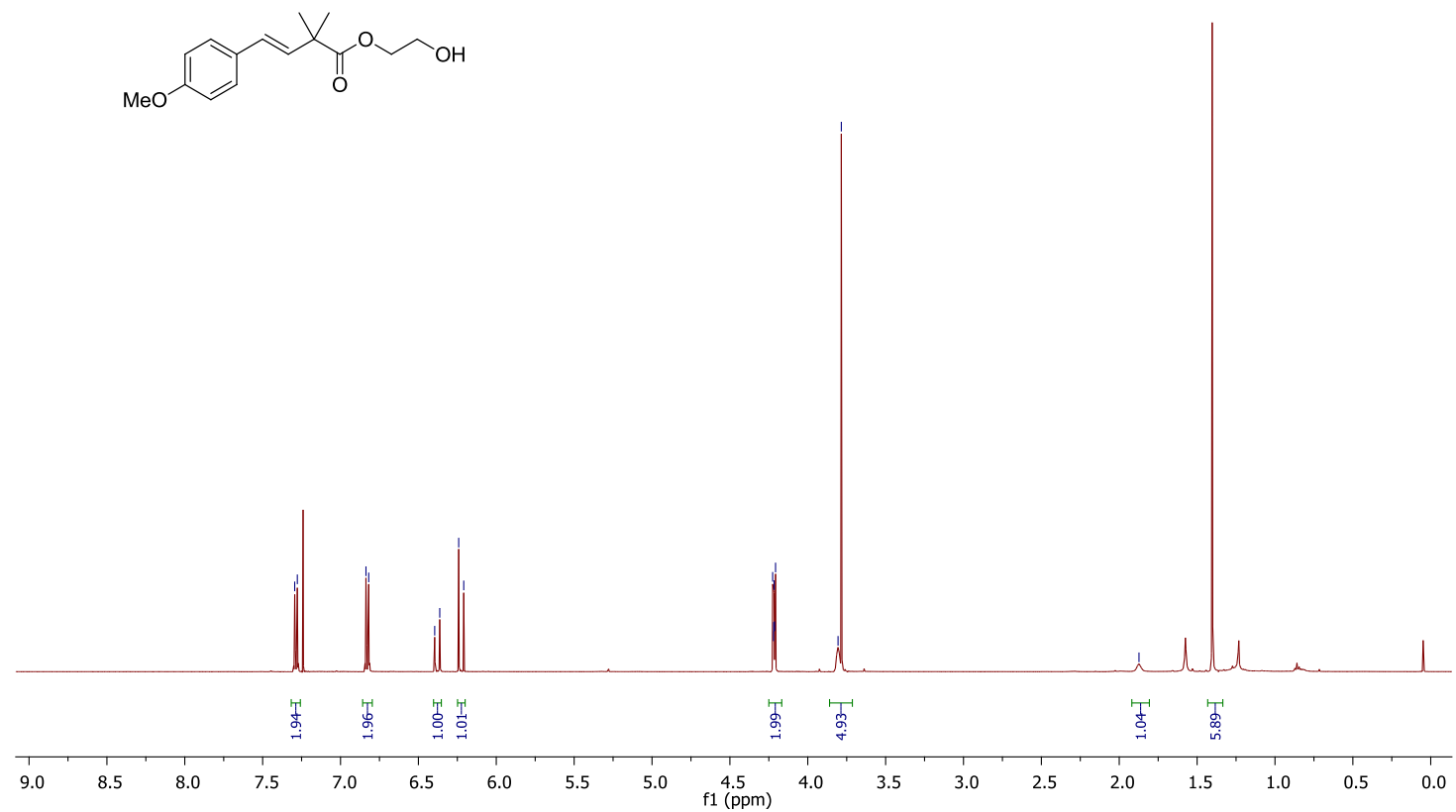

${ }^{13} \mathrm{C}\left\{{ }^{1} \mathrm{H}\right\}$ NMR $\left(126 \mathrm{MHz}, \mathrm{CDCl}_{3}\right)$

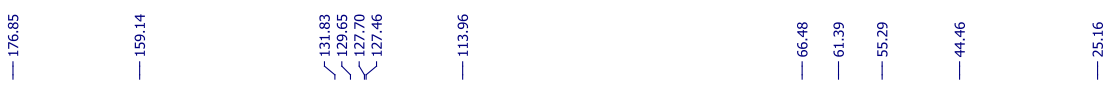

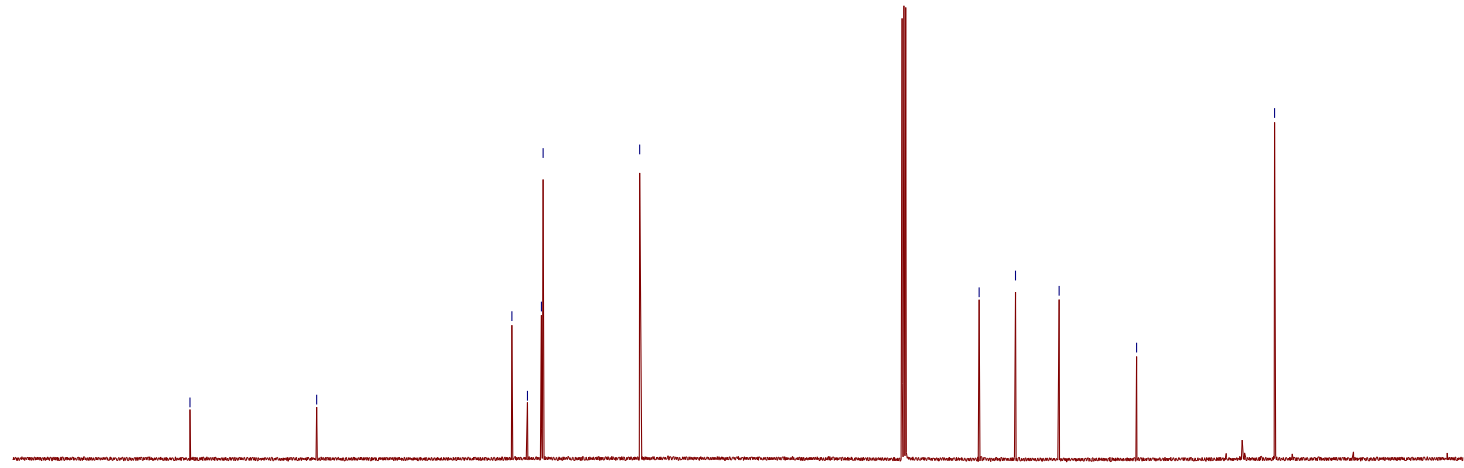

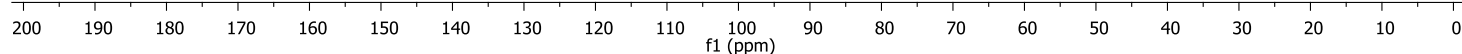


3f, [1284267-70-4]

${ }^{1} \mathrm{H}$ NMR (400 MHz, $\mathrm{CDCl}_{3}$ )

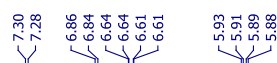

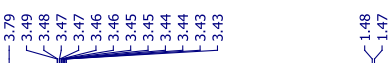
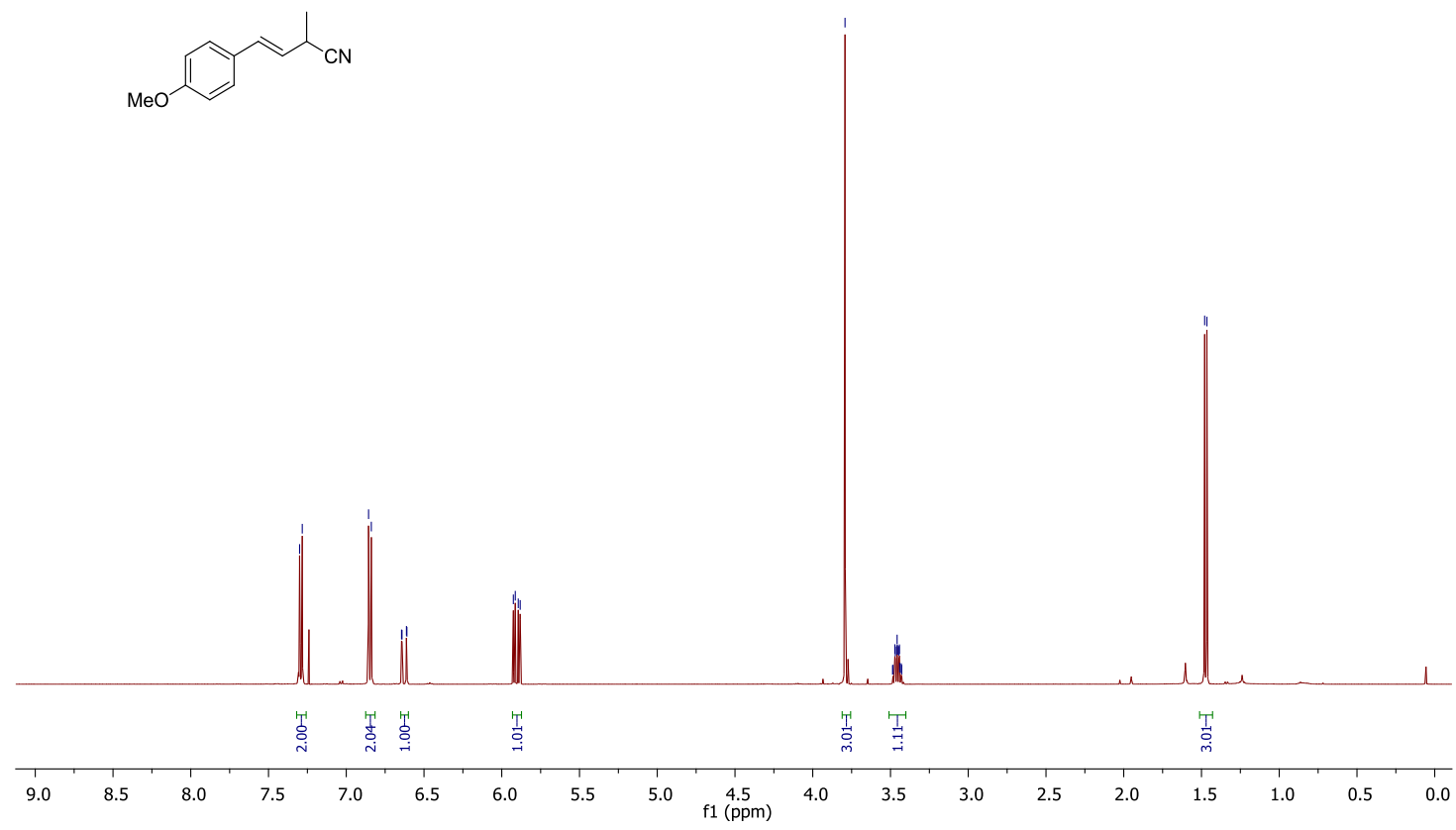

${ }^{13} \mathrm{C}\left\{{ }^{1} \mathrm{H}\right\} \mathrm{NMR}\left(126 \mathrm{MHz}, \mathrm{CDCl}_{3}\right)$

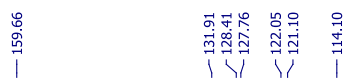

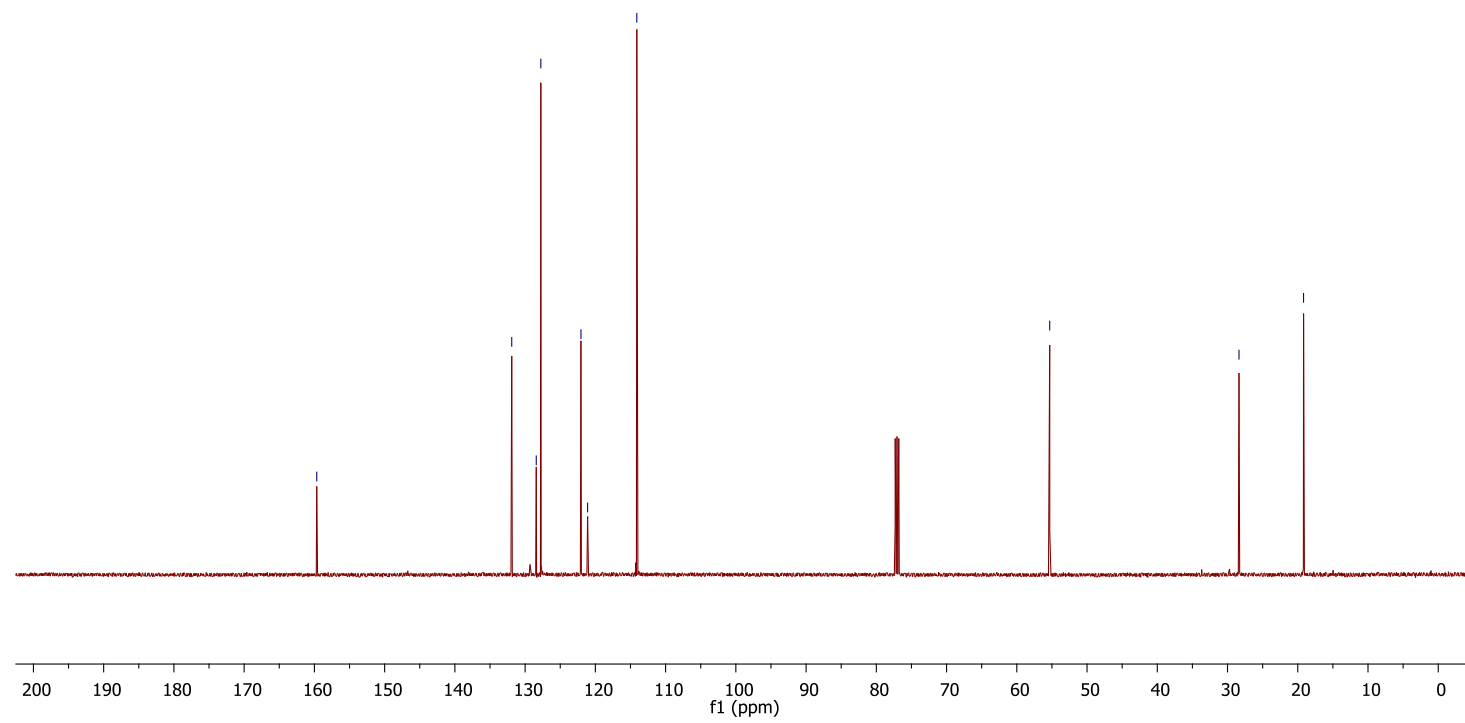


$3 g,[1276069-68-1]$

${ }^{1} \mathrm{H}$ NMR (400 MHz, $\mathrm{CDCl}_{3}$ )

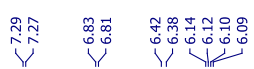

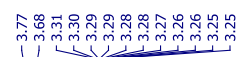

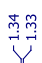

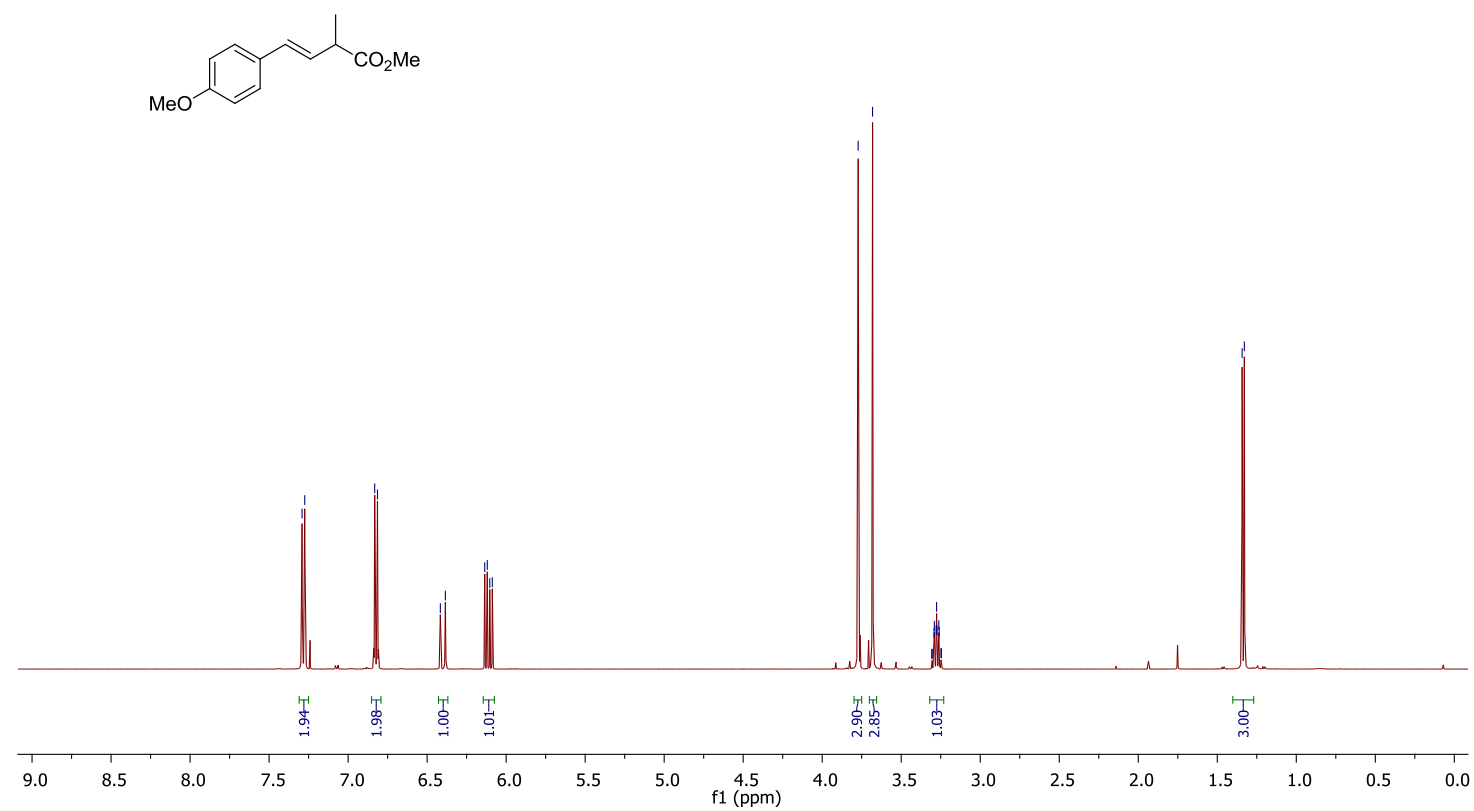

${ }^{13} \mathrm{C}\left\{{ }^{1} \mathrm{H}\right\}$ NMR $\left(126 \mathrm{MHz}, \mathrm{CDCl}_{3}\right)$

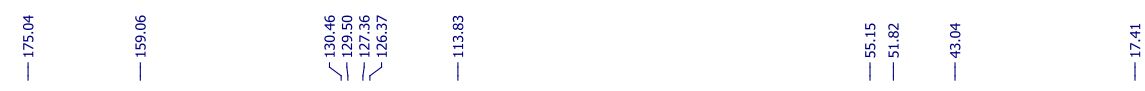

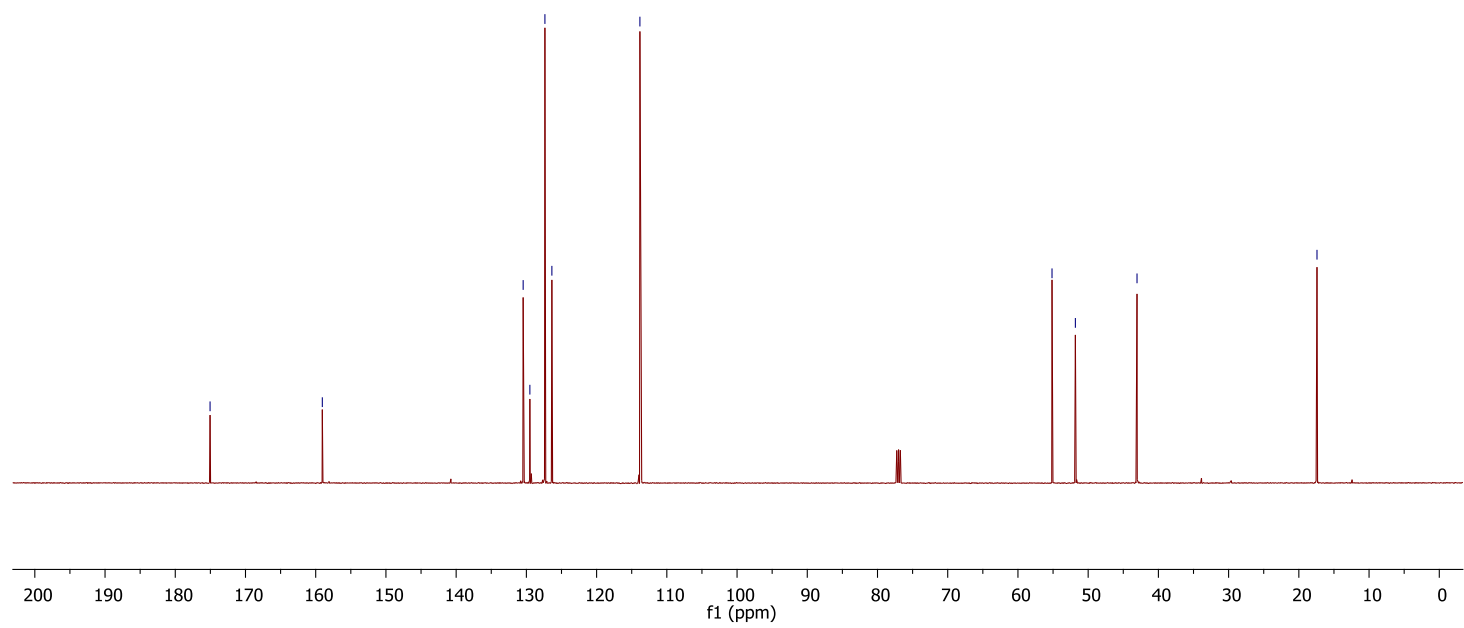


3h, [1627895-83-3]

${ }^{1} \mathrm{H}$ NMR (400 MHz, $\mathrm{CDCl}_{3}$ )

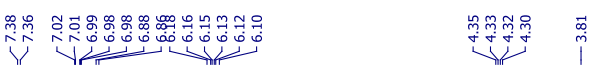

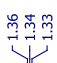

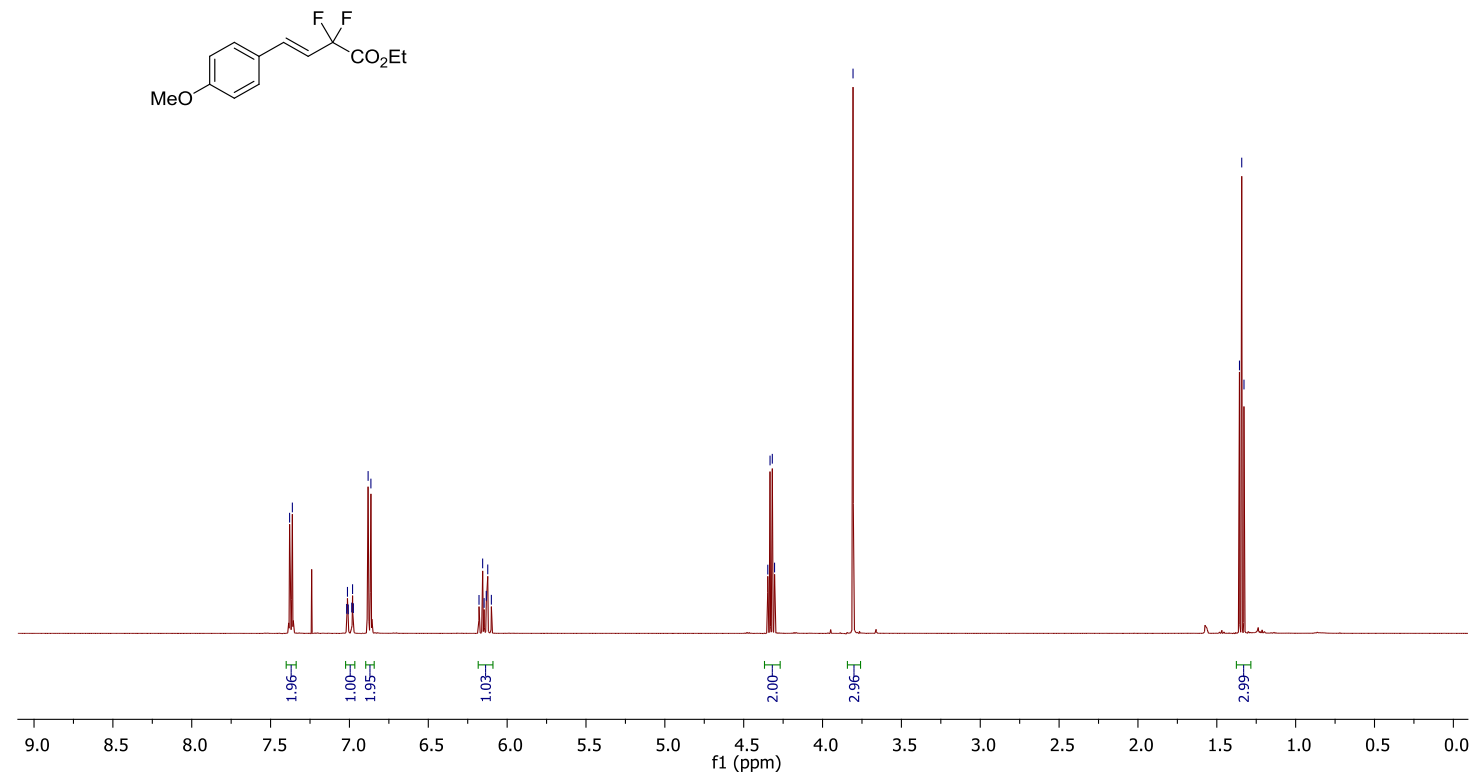

${ }^{13} \mathrm{C}\left\{{ }^{1} \mathrm{H}\right\}$ NMR $\left(126 \mathrm{MHz}, \mathrm{CDCl}_{3}\right)$

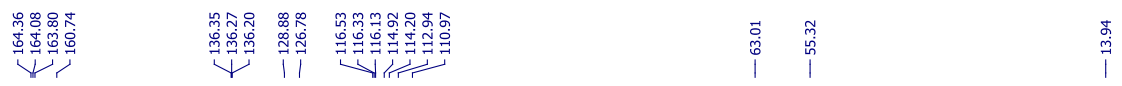

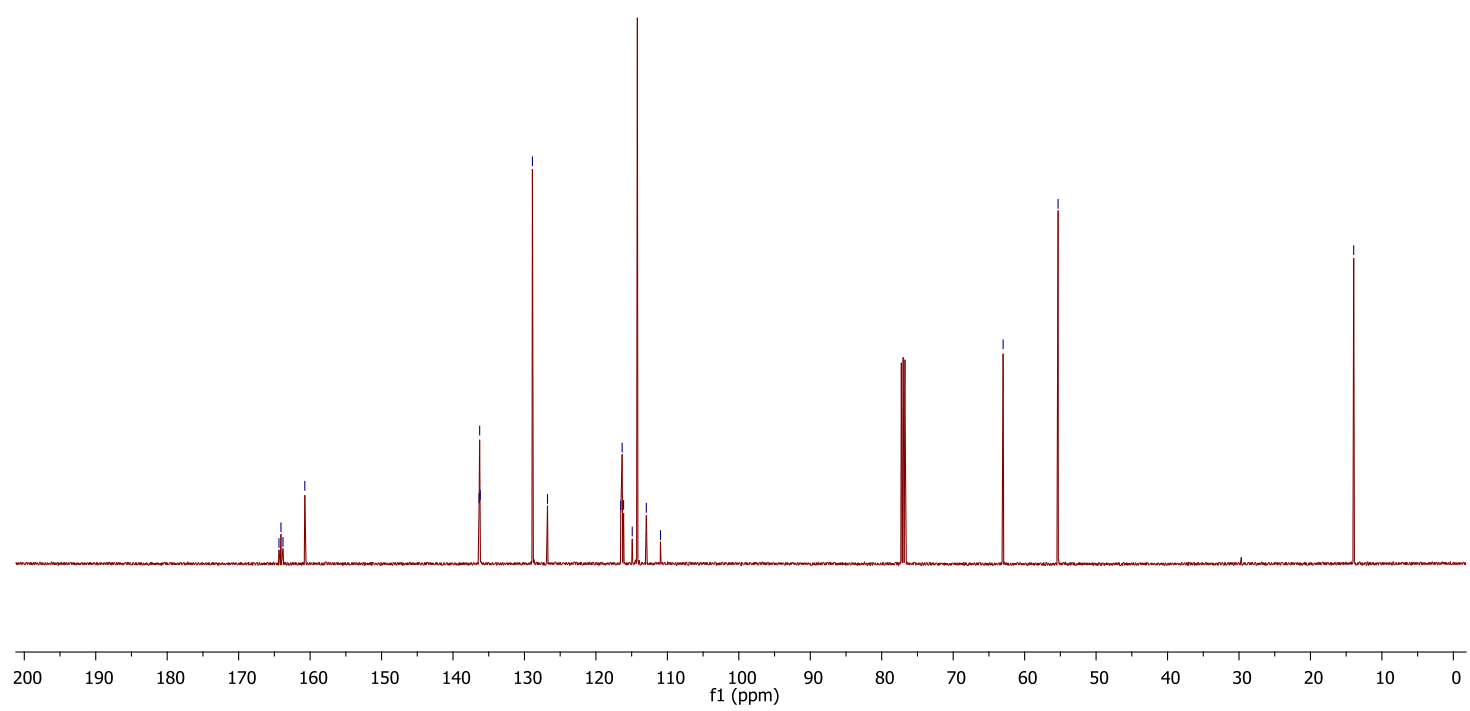


3i, [1433900-99-2]

${ }^{1} \mathrm{H}$ NMR (400 MHz, $\mathrm{CDCl}_{3}$ )

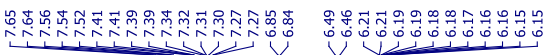
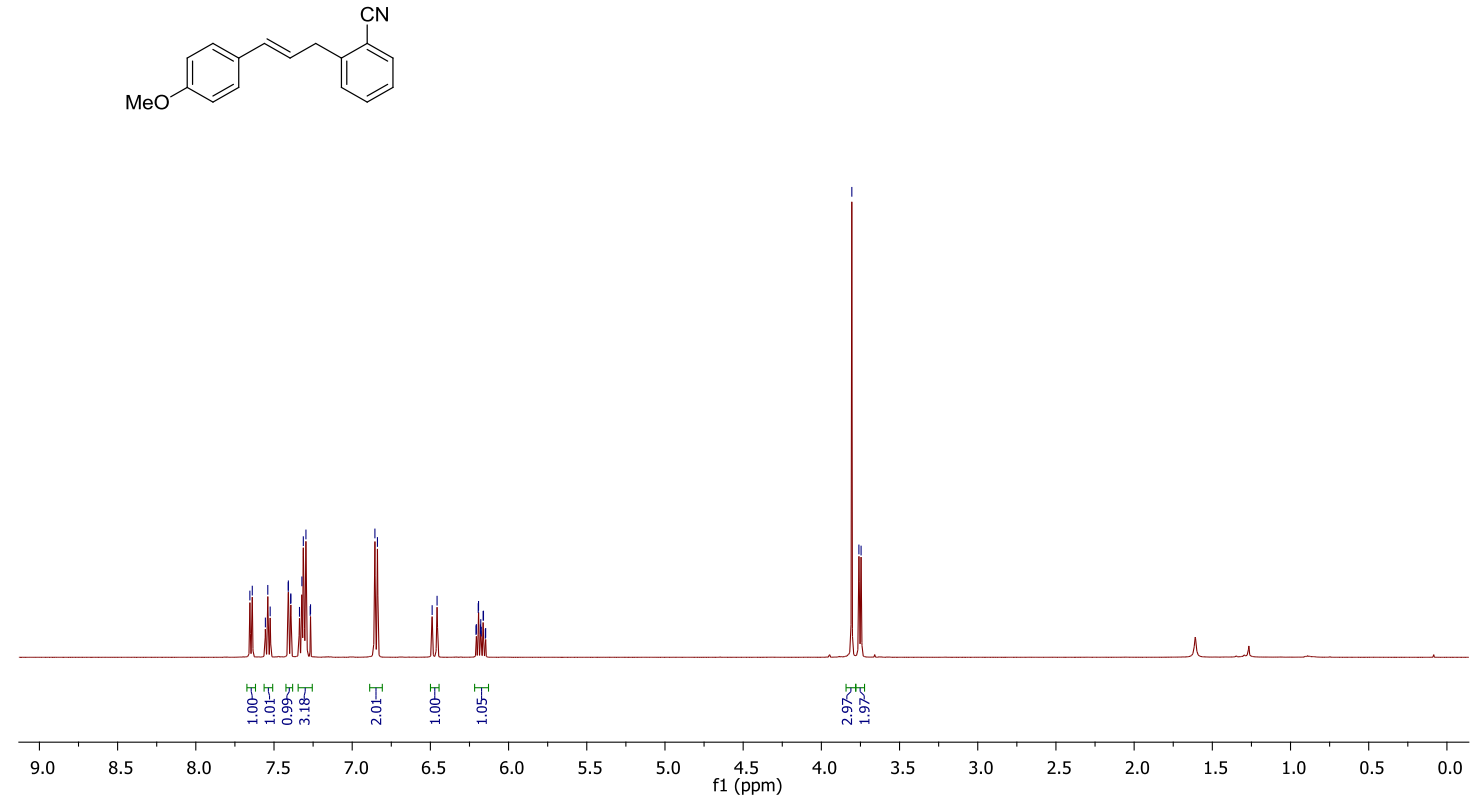

${ }^{13} \mathrm{C}\left\{{ }^{1} \mathrm{H}\right\}$ NMR $\left(126 \mathrm{MHz}, \mathrm{CDCl}_{3}\right)$

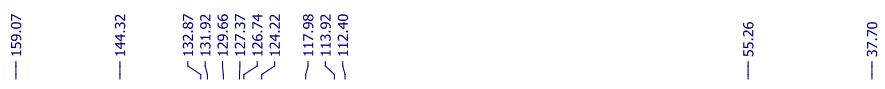

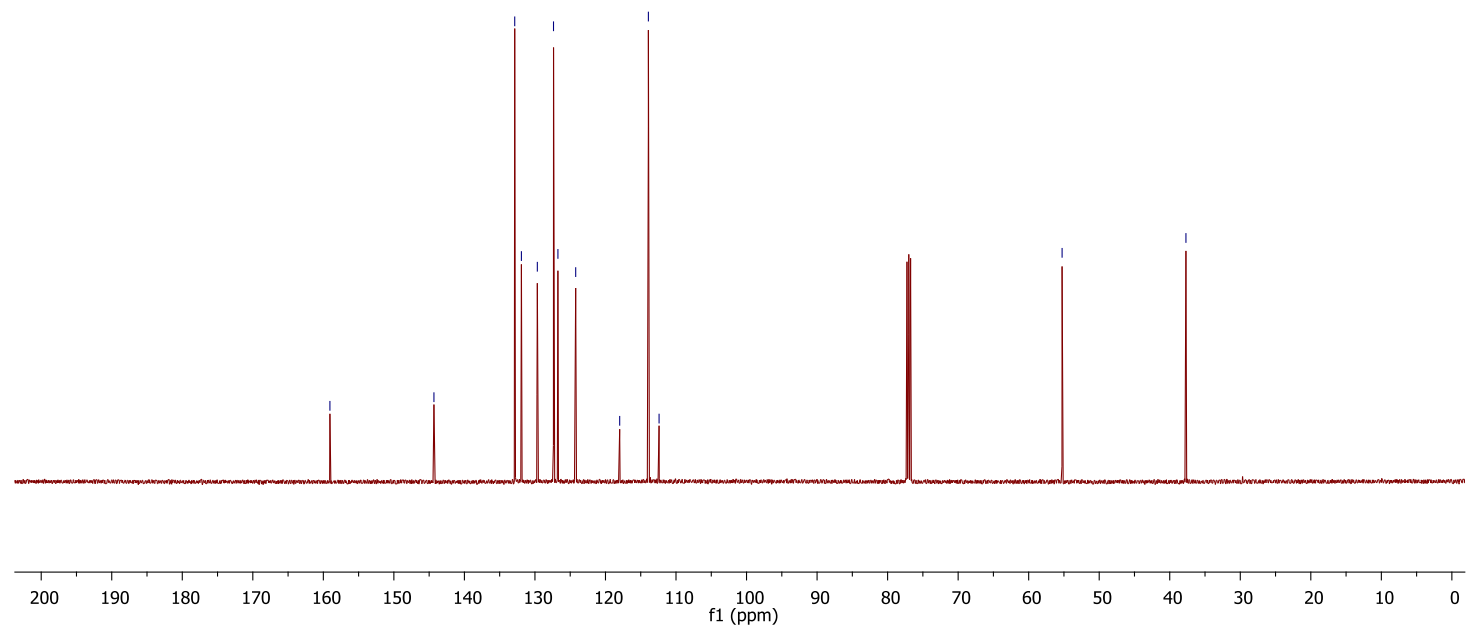


3j, [1289638-89-6]

${ }^{1} \mathrm{H}$ NMR (400 MHz, $\mathrm{CDCl}_{3}$ )

稌

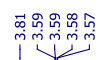
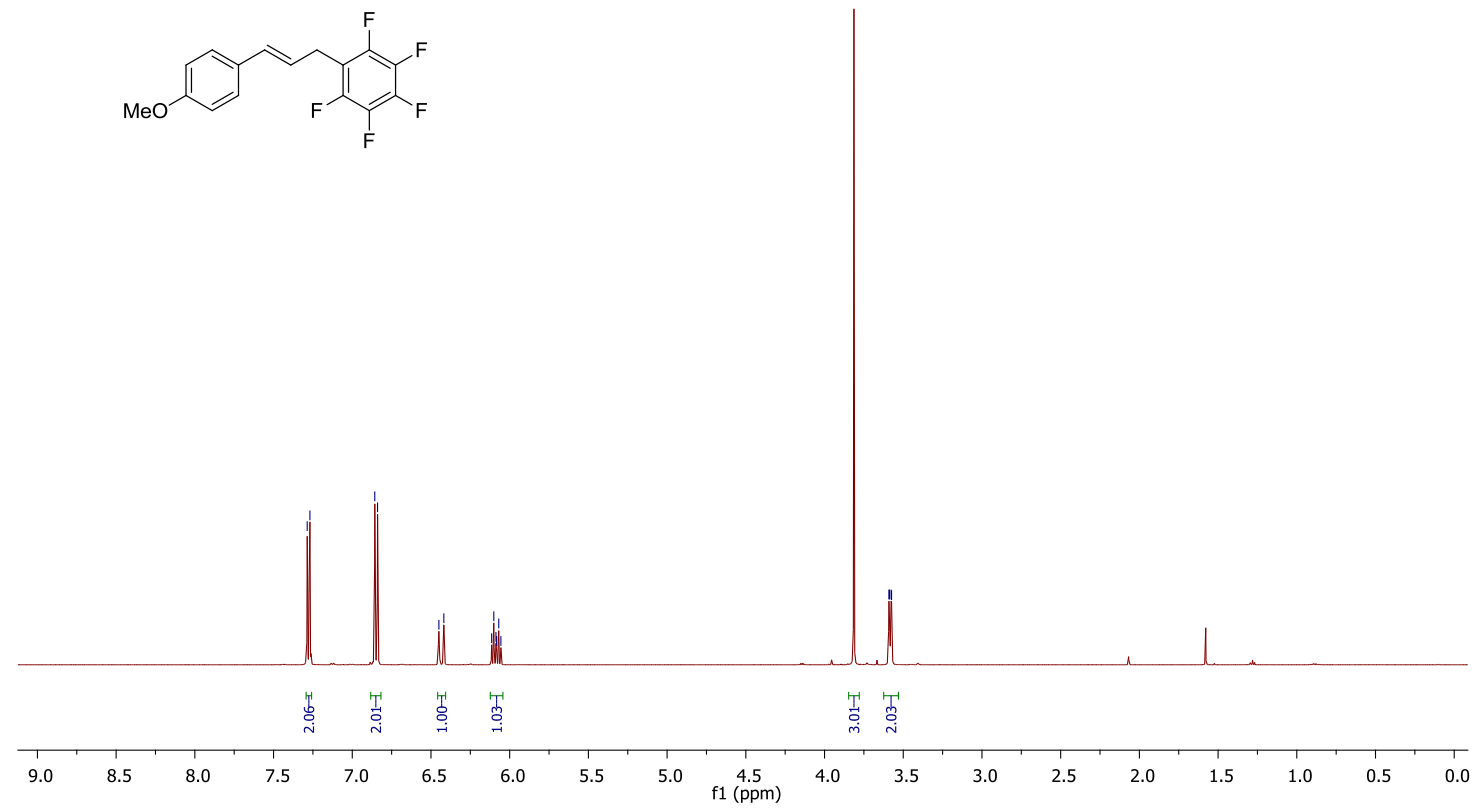

${ }^{13} \mathrm{C}\left\{{ }^{1} \mathrm{H}\right\}$ NMR $\left(126 \mathrm{MHz}, \mathrm{CDCl}_{3}\right)$

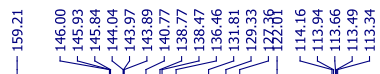

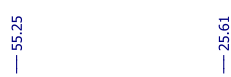

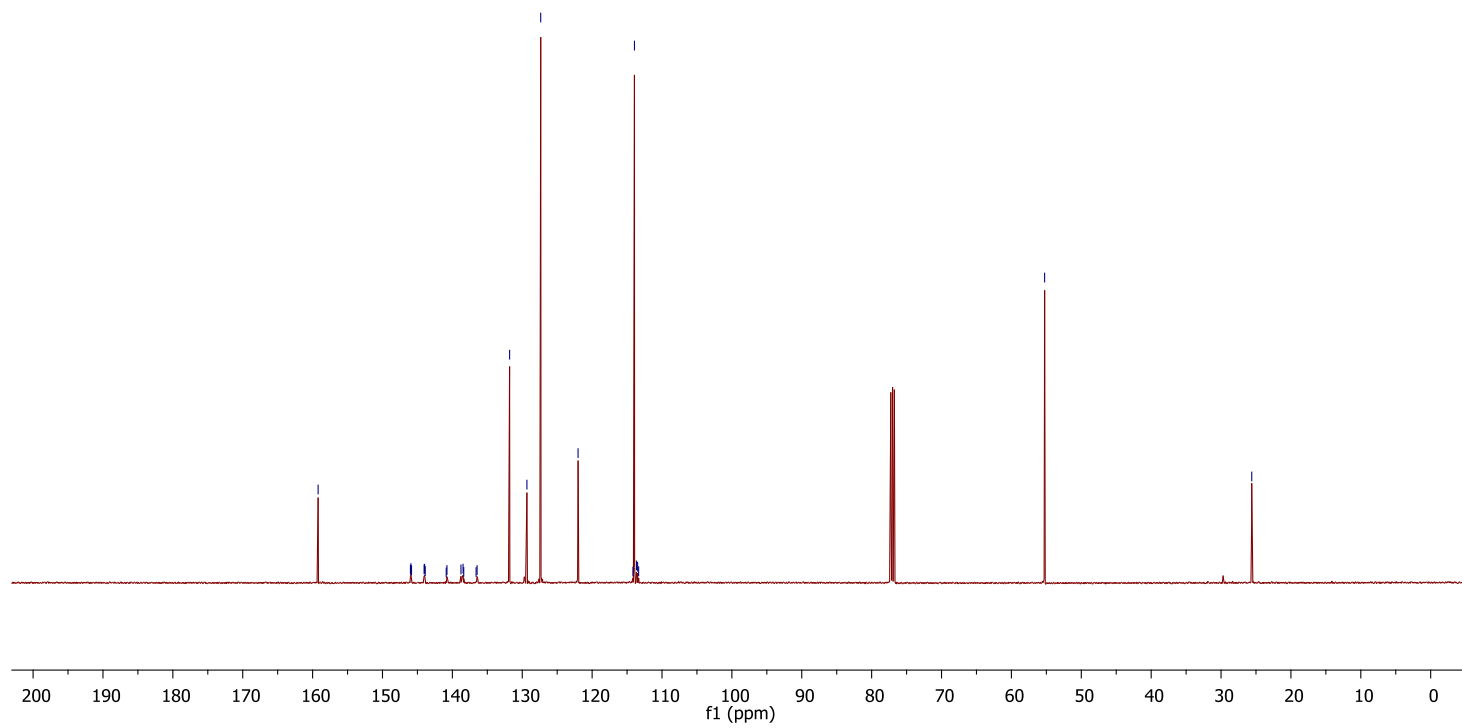


3k, [1380341-73-0]

${ }^{1} \mathrm{H}$ NMR $\left(400 \mathrm{MHz}, \mathrm{CDCl}_{3}\right)$

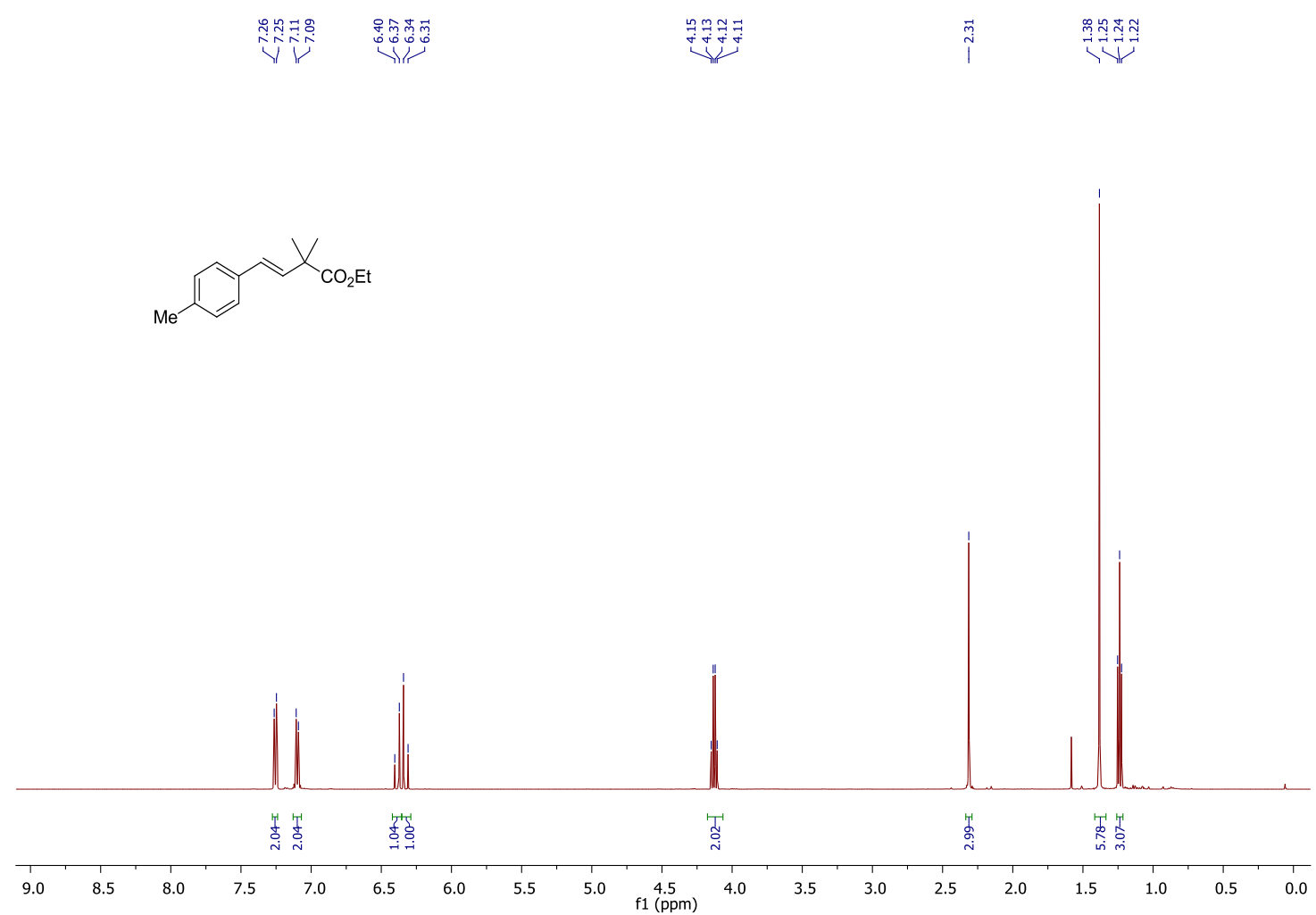

${ }^{13} \mathrm{C}\left\{{ }^{1} \mathrm{H}\right\} \mathrm{NMR}(126 \mathrm{MHz}, \mathrm{CDCl})$

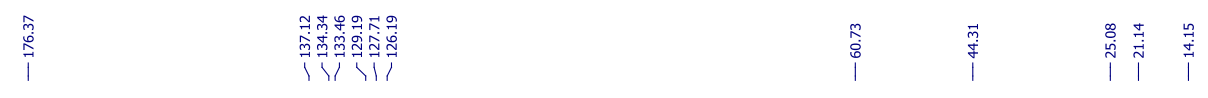

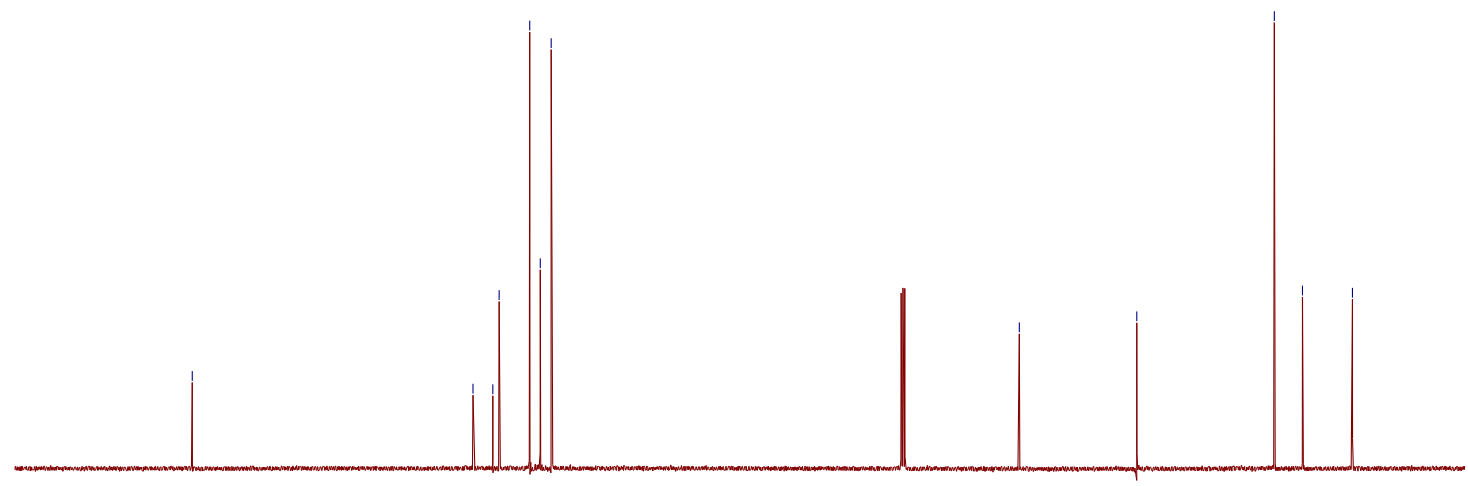

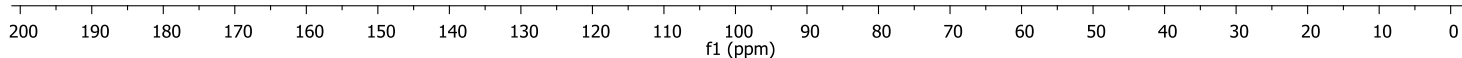


3I

${ }^{1} \mathrm{H}$ NMR $\left(400 \mathrm{MHz}, \mathrm{CDCl}_{3}\right)$

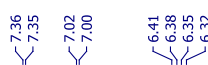

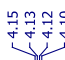

กิ
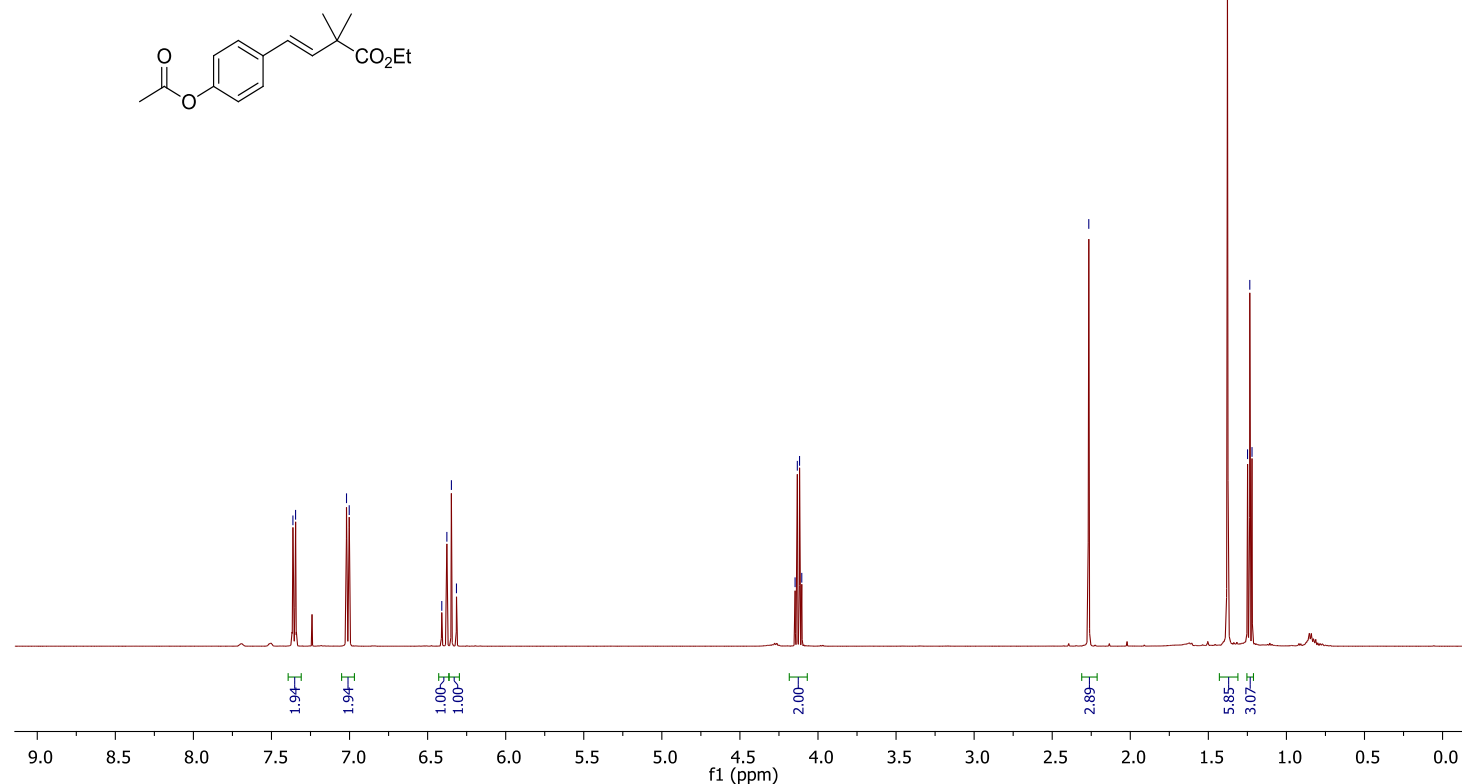

${ }^{13} \mathrm{C}\left\{{ }^{1} \mathrm{H}\right\}$ NMR $\left(126 \mathrm{MHz}, \mathrm{CDCl}_{3}\right)$

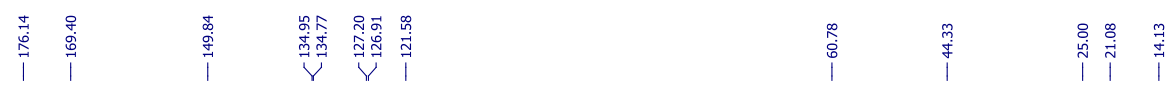

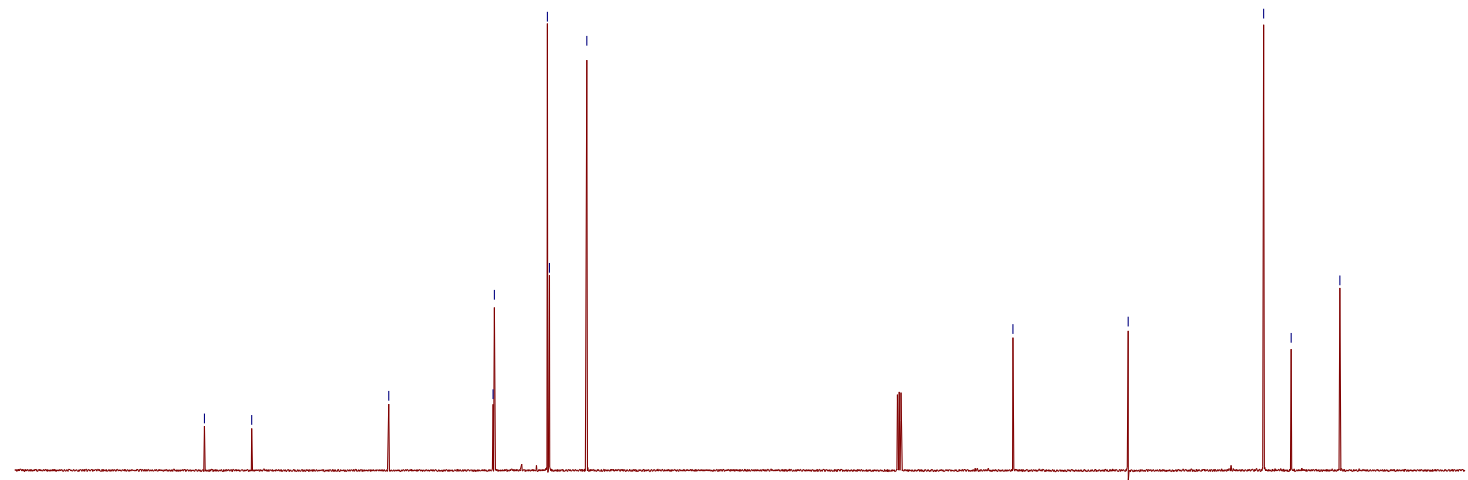

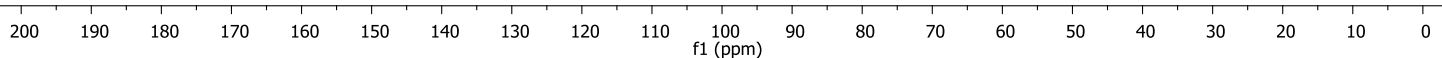


3m, [2086695-44-3]

${ }^{1} \mathrm{H}$ NMR (400 MHz, $\mathrm{CDCl}_{3}$ )

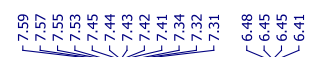

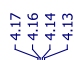

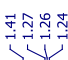

$X_{\mathrm{CO}_{2} \mathrm{Et}}$

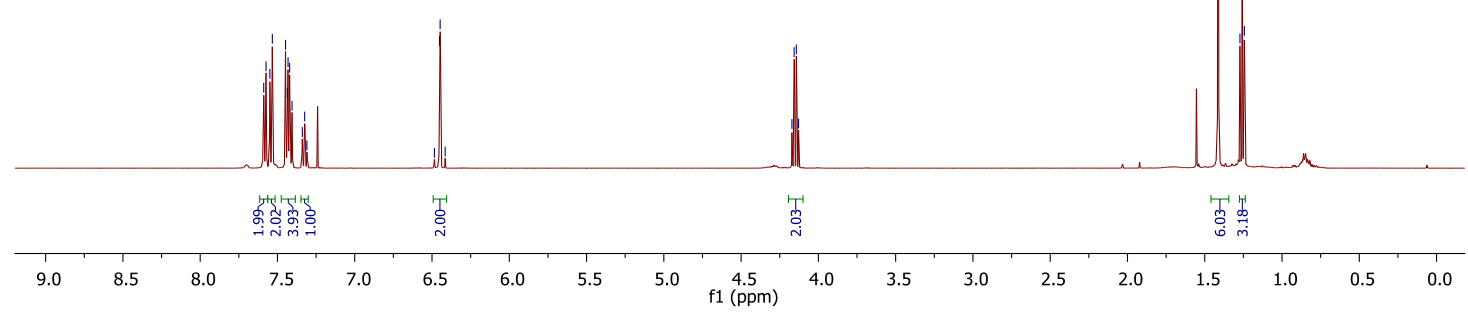

${ }^{13} \mathrm{C}\left\{{ }^{1} \mathrm{H}\right\}$ NMR $\left(126 \mathrm{MHz}, \mathrm{CDCl}_{3}\right)$

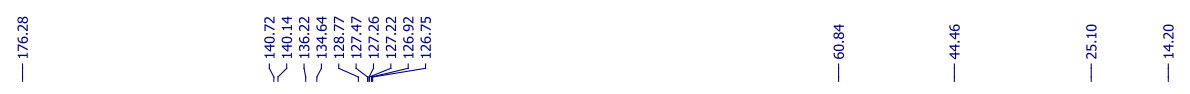

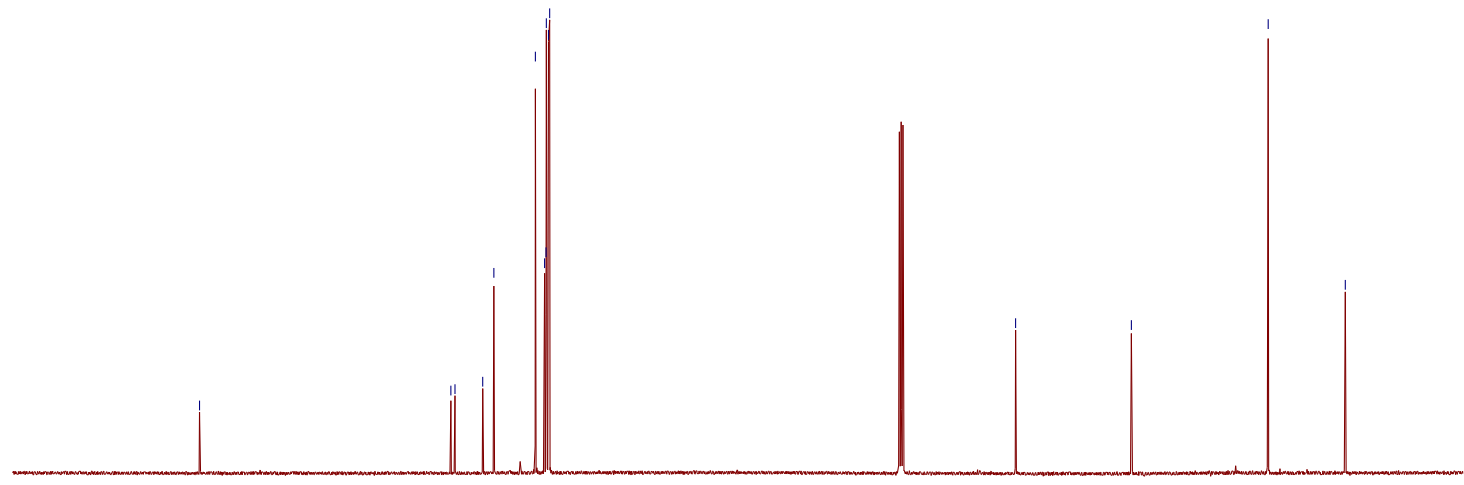

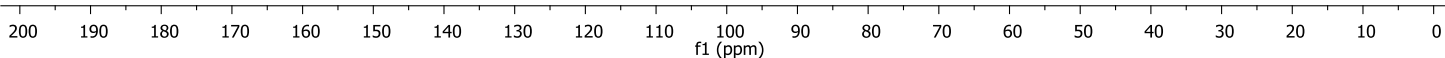


3n, [1380341-75-2]

${ }^{1} \mathrm{H}$ NMR (400 MHz, $\mathrm{CDCl}_{3}$ )

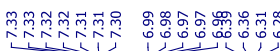

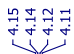

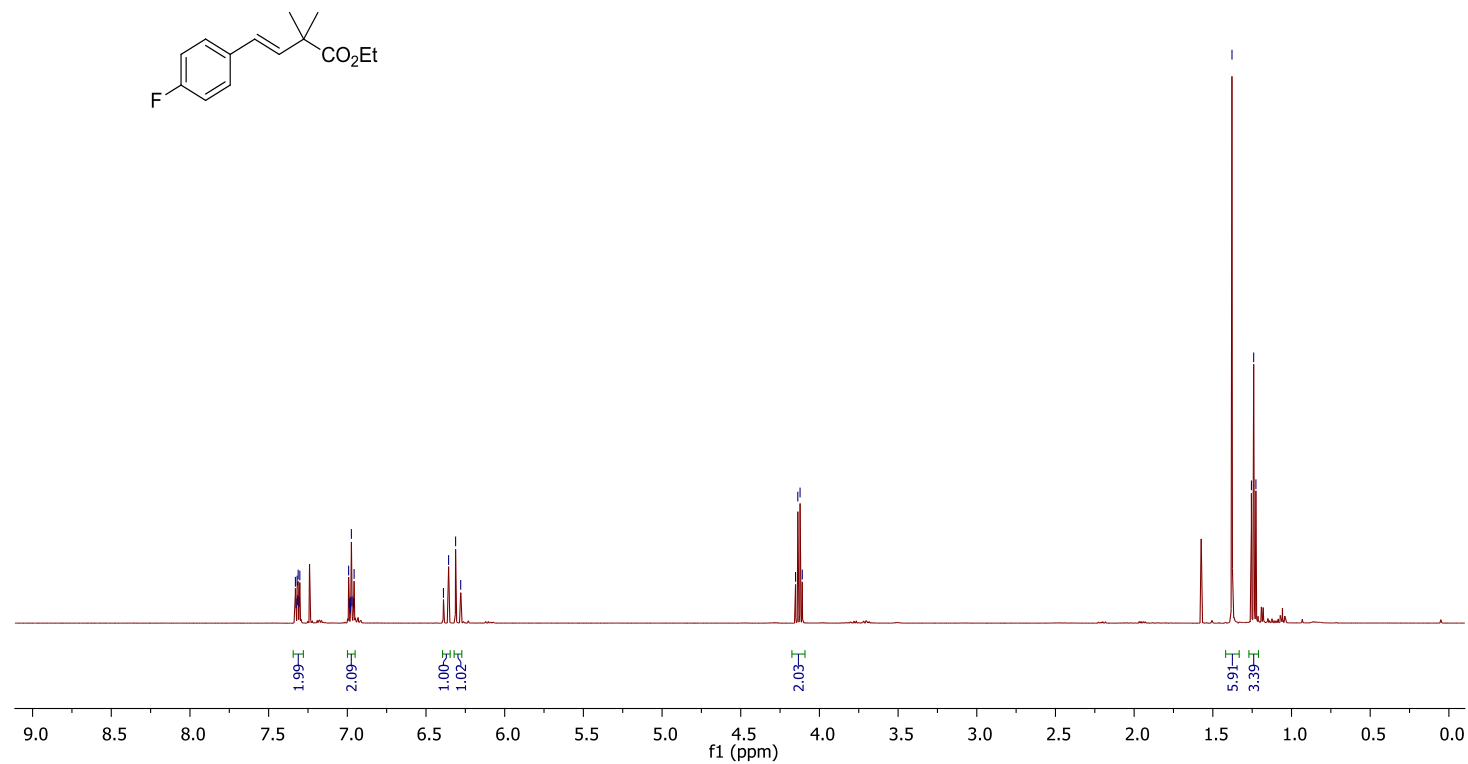

${ }^{13} \mathrm{C}\left\{{ }^{1} \mathrm{H}\right\}$ NMR $\left(126 \mathrm{MHz}, \mathrm{CDCl}_{3}\right)$

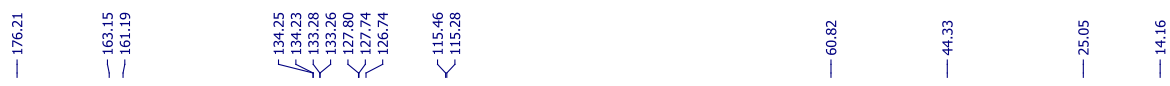

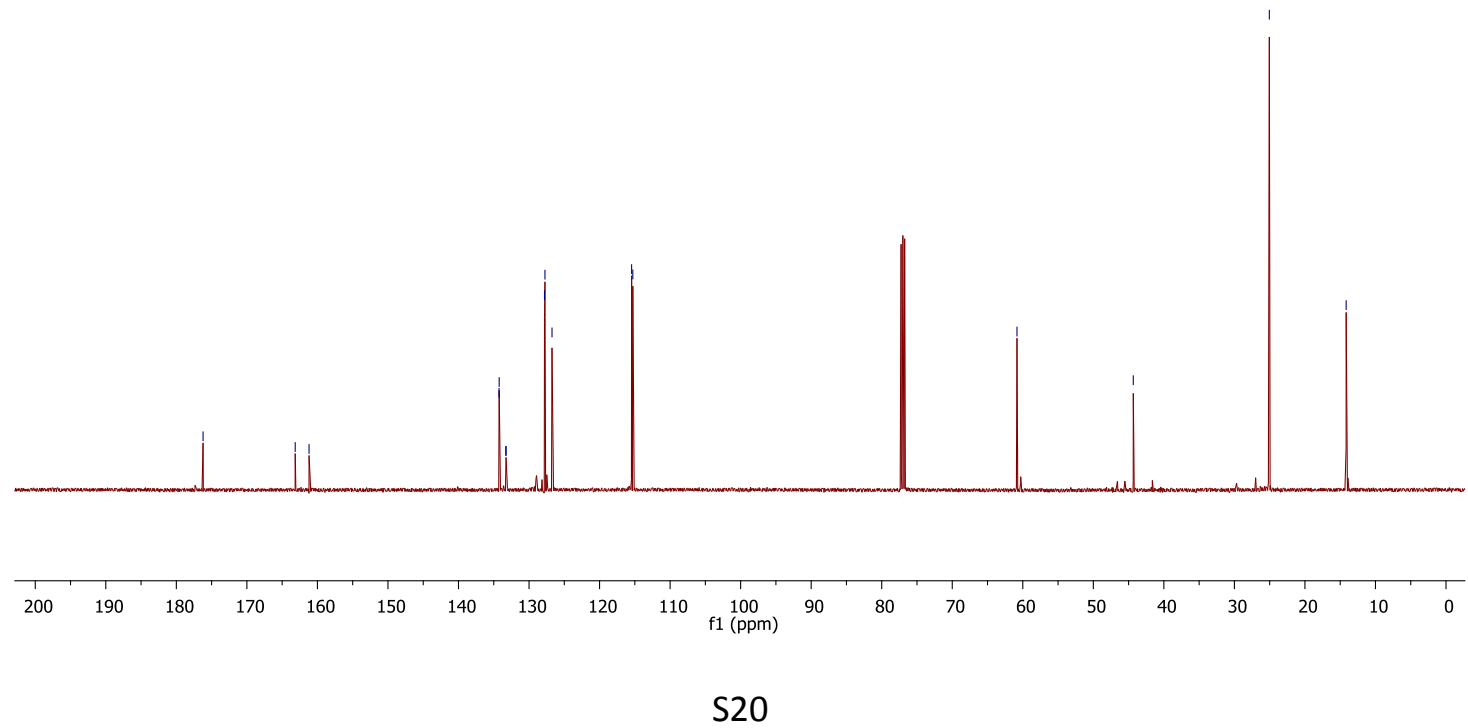


30

${ }^{1} \mathrm{H}$ NMR (400 MHz, $\mathrm{CDCl}_{3}$ )

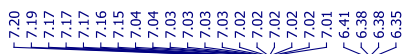

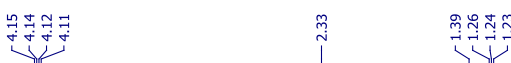

$X_{\mathrm{CO}_{2} \mathrm{Et}}$

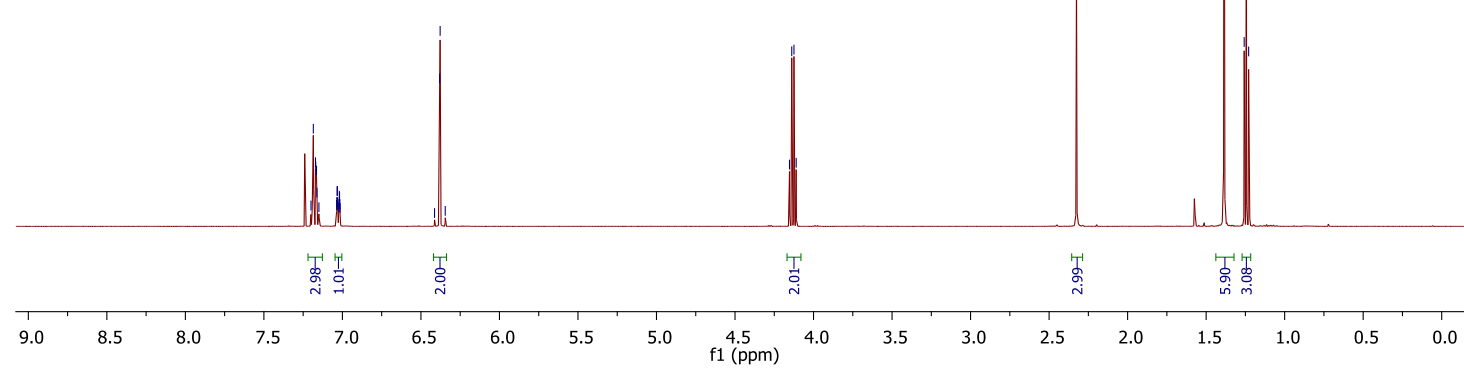

${ }^{13} \mathrm{C}\left\{{ }^{1} \mathrm{H}\right\}$ NMR $\left(126 \mathrm{MHz}, \mathrm{CDCl}_{3}\right)$

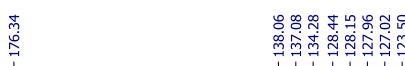

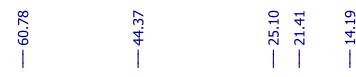

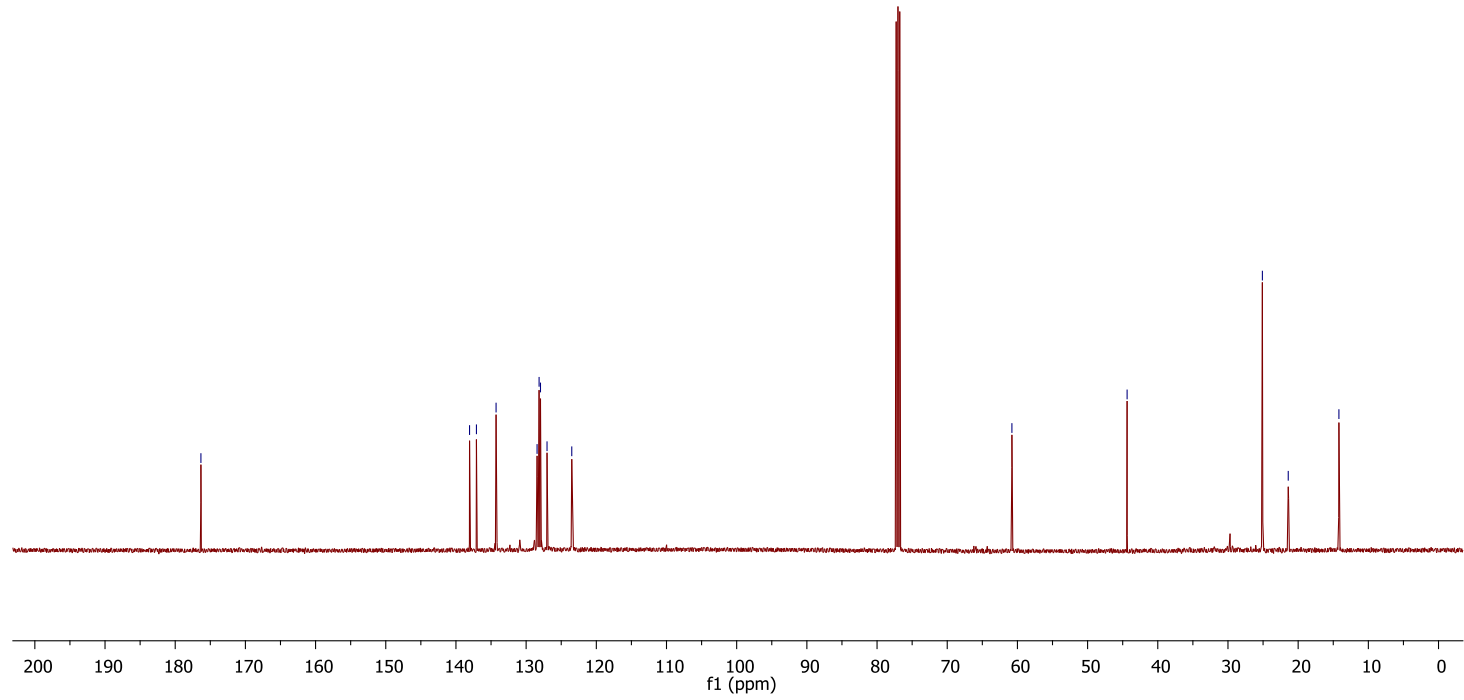


$3 p$

${ }^{1} \mathrm{H}$ NMR $\left(400 \mathrm{MHz}, \mathrm{CDCl}_{3}\right)$

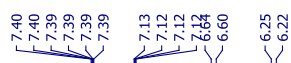

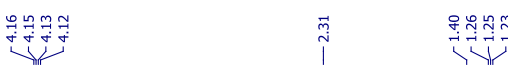
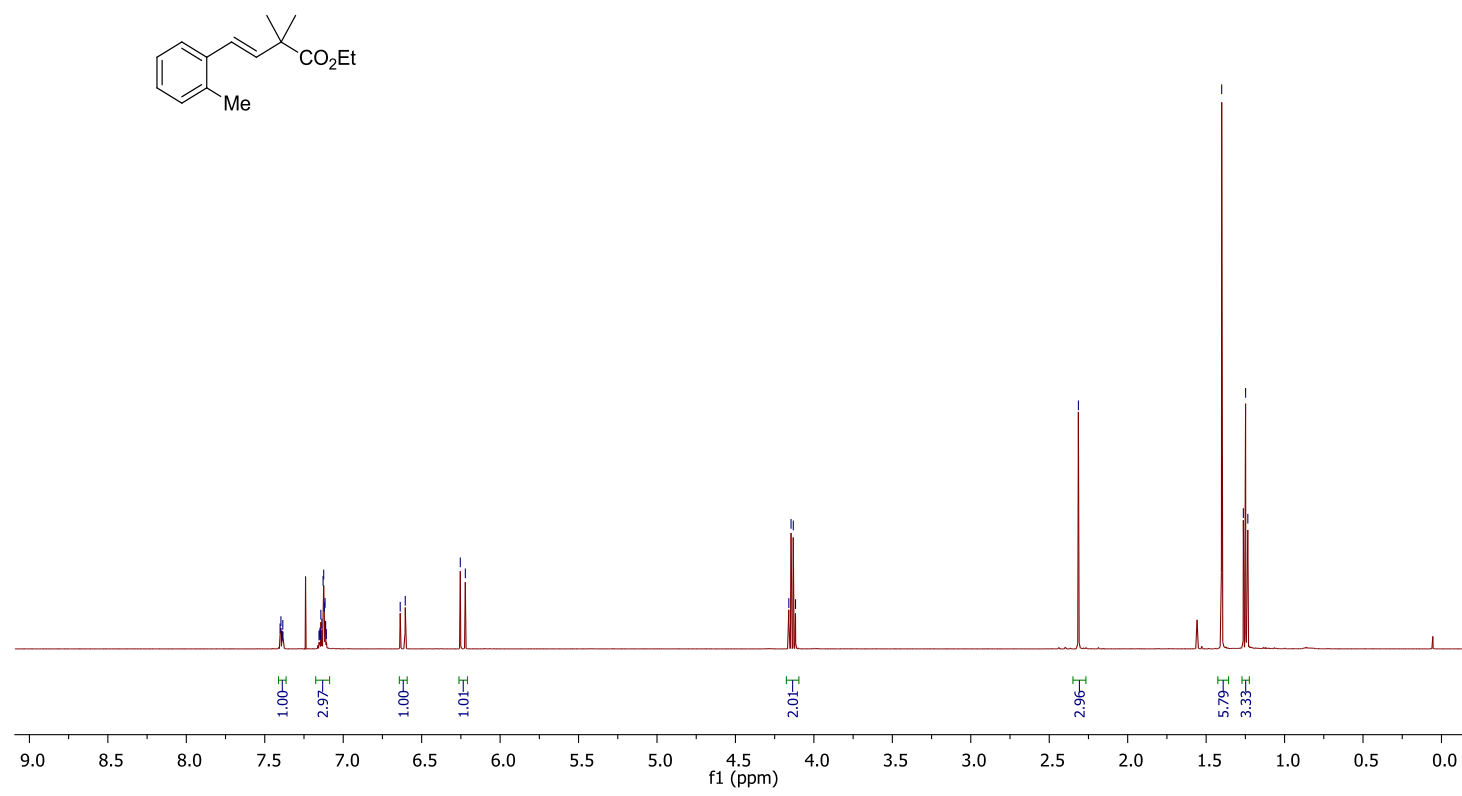

${ }^{13} \mathrm{C}\left\{{ }^{1} \mathrm{H}\right\}$ NMR $\left(126 \mathrm{MHz}, \mathrm{CDCl}_{3}\right)$

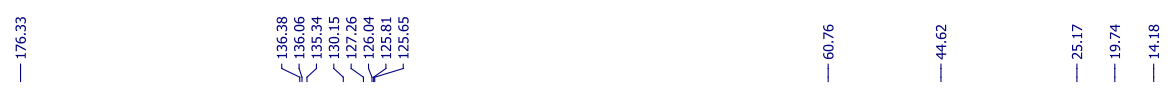

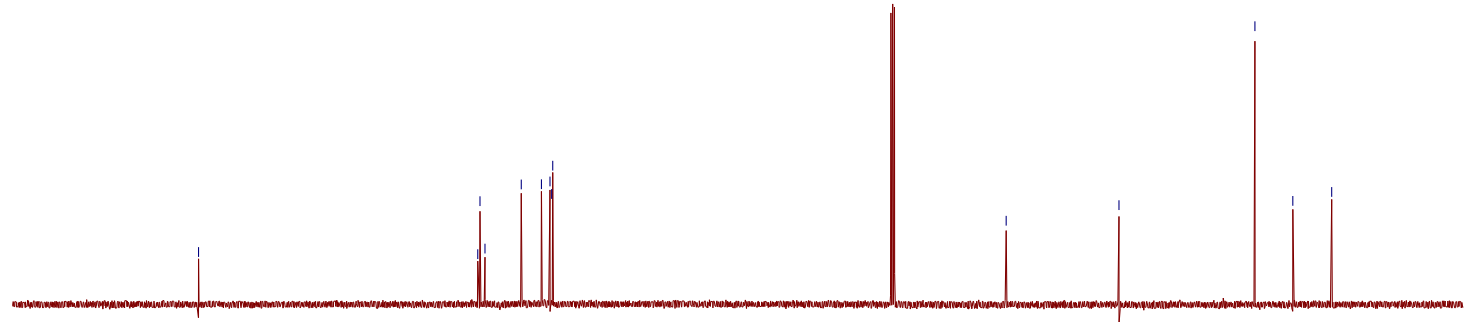

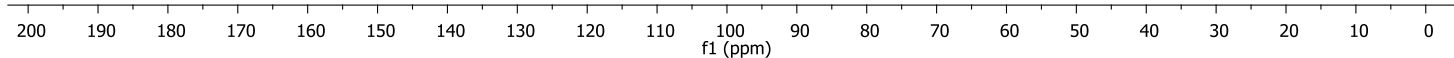


3r, [158987-27-0]

${ }^{1} \mathrm{H}$ NMR $\left(400 \mathrm{MHz}, \mathrm{CDCl}_{3}\right)$

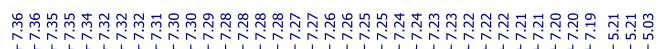

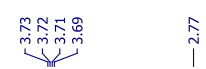

iri

$\mathrm{X}_{\mathrm{CO}_{2} \mathrm{Et}}$

${ }^{13} \mathrm{C}\left\{{ }^{1} \mathrm{H}\right\}$ NMR $\left(126 \mathrm{MHz}, \mathrm{CDCl}_{3}\right)$

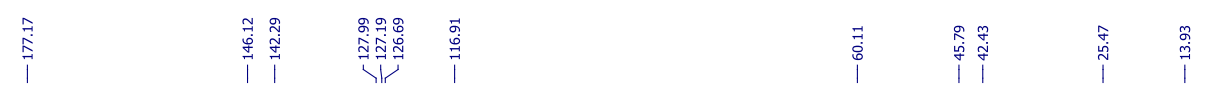

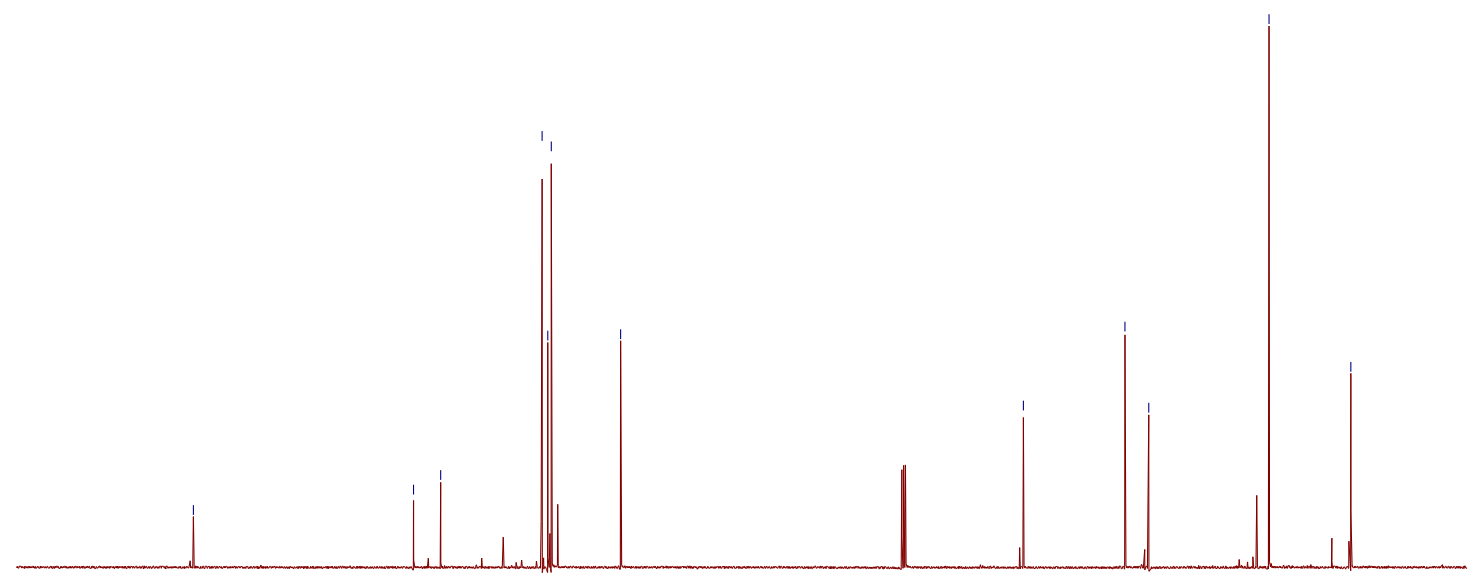

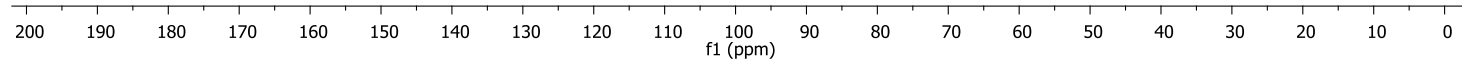


4, [212128-91-1]

${ }^{1} \mathrm{H}$ NMR $\left(400 \mathrm{MHz}, \mathrm{CDCl}_{3}\right)$

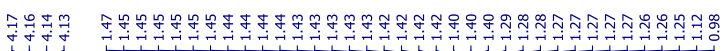

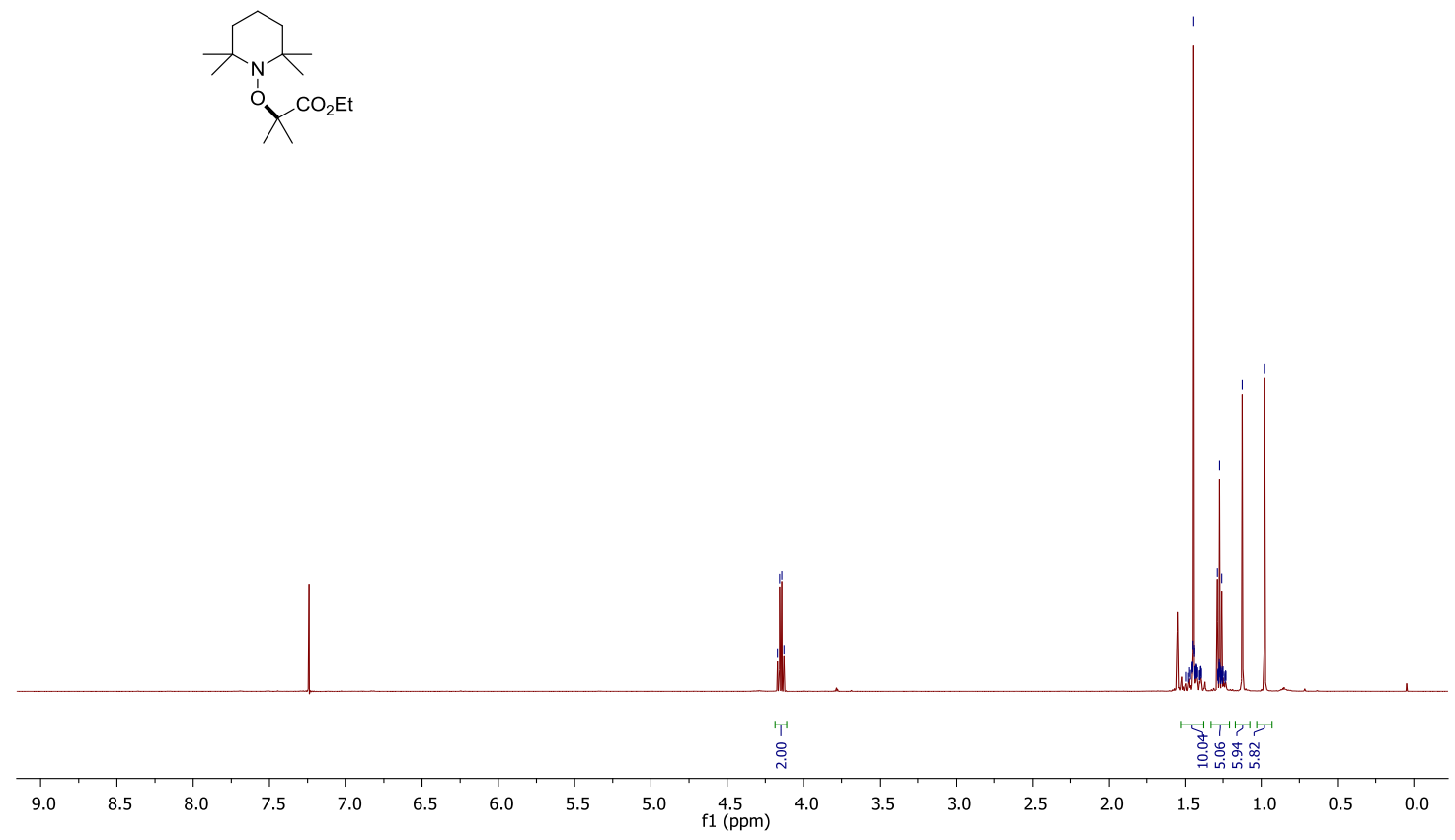

${ }^{13} \mathrm{C}\left\{{ }^{1} \mathrm{H}\right\} \mathrm{NMR}\left(126 \mathrm{MHz}, \mathrm{CDCl}_{3}\right)$
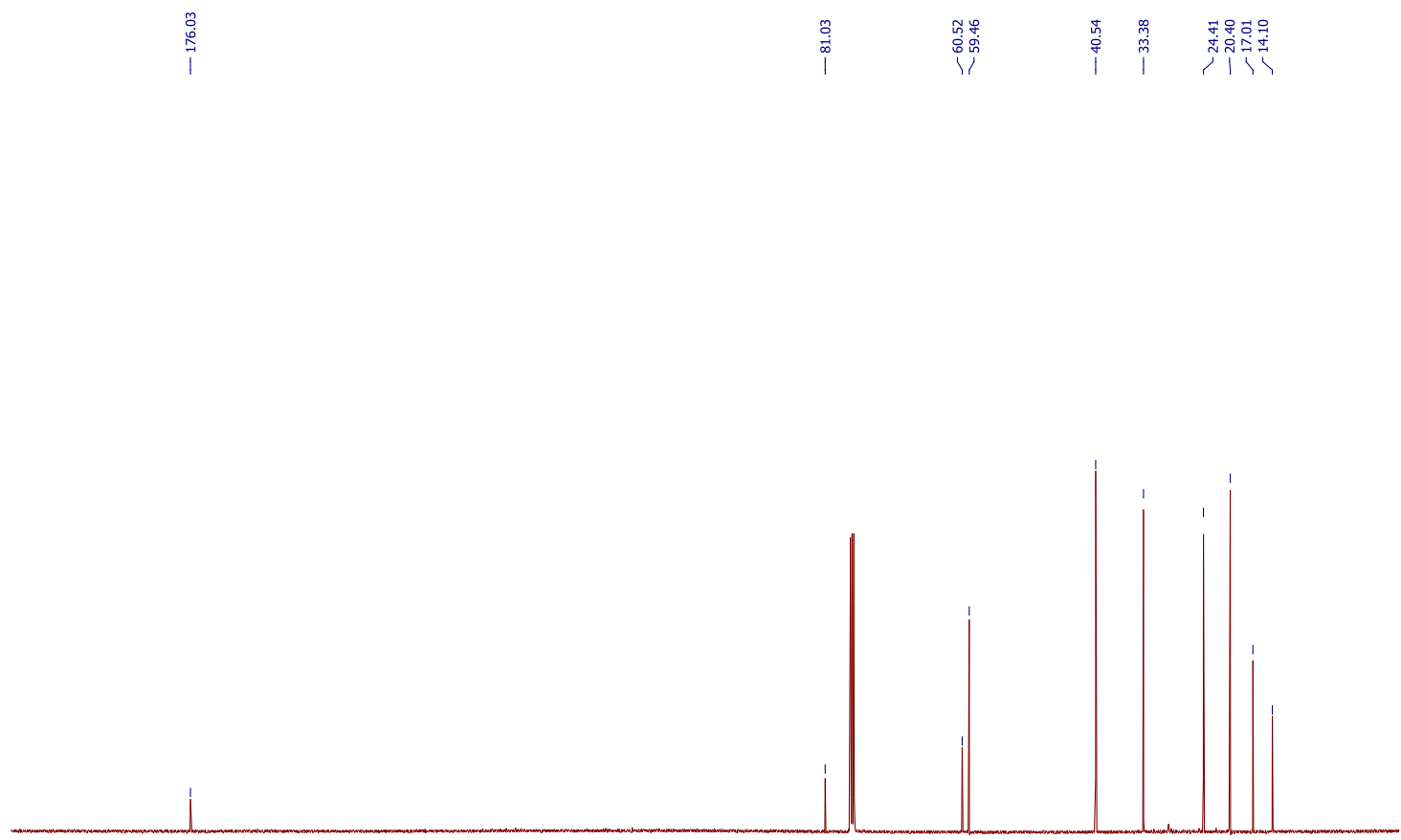

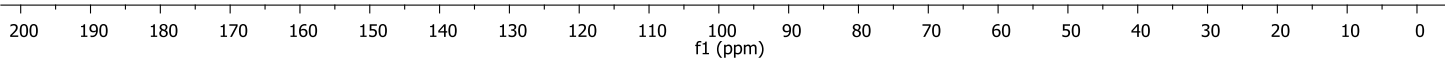




\section{X-ray crystallographic data for $\mathbf{3 d}$}

Single crystal of suitable size was covered with perfluoroether oil (Fomblin Y) and mounted on a MITIGEN holder. Data collection has been performed on a Bruker D8 QUEST diffractometer equipped with a Photon100 CMOS detector, using MoK $\alpha$ radiation ( $\lambda=0.71073 \AA$, microfocus sealed $x$-ray tube) and an Oxford Cryosystems lowtemperature device (Cryostream 800).

Data collection was processed with APEX3 Suite (Bruker, v.2017), cell refinement and data reduction with SAINT (Bruker, v8.38A). A multi-scan absorption correction was performed using SADABS-2016/2.

The structure was solved by intrinsic phasing method using the XT (Sheldrick, 2015) ${ }^{1}$ structure solution program and refined using full matrix least squares on $\mathrm{F}^{2}$ minimisation using XL-2016/6 (Sheldrick, 2014) ${ }^{2}$ and molecular graphics software Olex2 package (Dolomanov et al., 2009). ${ }^{3}$ Non-hydrogen atoms were refined with anisotropic displacement parameters and hydrogen atoms attached to refined atoms were placed in geometrically idealized positions and refined by using a riding model.

A summary of the fundamental crystal and refinement data are given in the Table 1 of the Supporting Information. Atomic coordinates, anisotropic displacement parameters and bond lengths and angles can be found in the cif files. Crystallographic data has been deposited in the Cambridge Crystallographic Data Centre with no. 1899012.

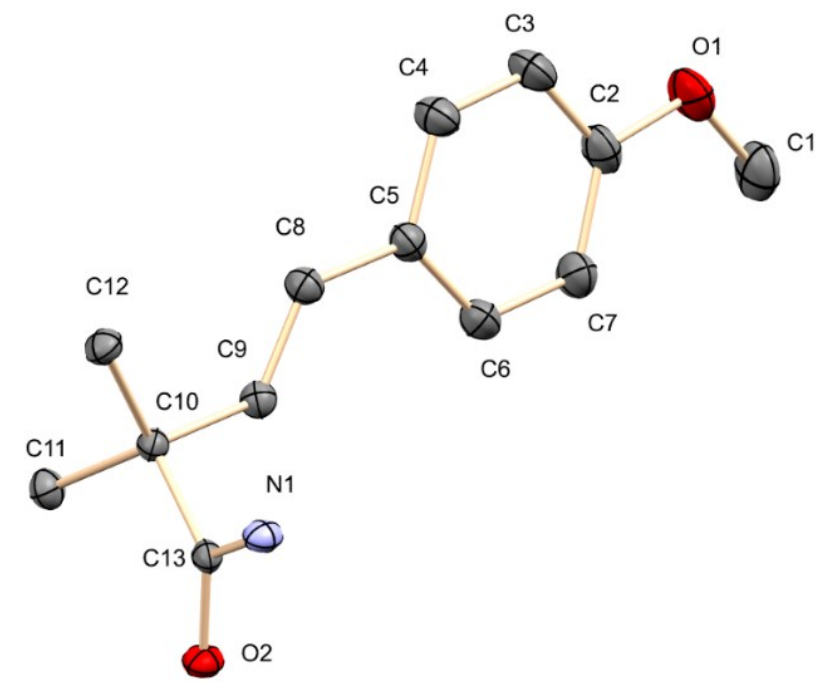

ORTEP view of the molecules of complex $\mathbf{3 d}$, showing ellipsoids at $30 \%$ probability level.

Table 1. Crystal data and structure refinement for compound 3d.

Identification code

Empirical formula

Formula weight 180613p117_0ma_a

$\mathrm{C} 13 \mathrm{H} 17 \mathrm{~N} \mathrm{O} 2$

219.27 
Temperature

Wavelength

Crystal system

Space group

Unit cell dimensions

Volume

Z

Density (calculated)

Absorption coefficient

$\mathrm{F}(000)$

Crystal size

Theta range for data collection

Index ranges

Reflections collected

Independent reflections

Completeness to theta $=25.242^{\circ}$

Absorption correction

Max. and min. transmission

Refinement method

Data / restraints / parameters

Goodness-of-fit on $F^{2}$

Final $R$ indices [ $>2$ sigma(I)]

$R$ indices (all data)

Extinction coefficient

Largest diff. peak and hole
$100.0 \mathrm{~K}$

$0.71073 \AA$

Monoclinic

$\mathrm{P} 21 / \mathrm{c}$

$a=20.5410(12) \AA \quad \alpha=90^{\circ}$.

$\mathrm{b}=5.8794(4) \AA$

$c=10.1091(6) \AA$

$1203.24(13) \AA^{3}$

4

$1.210 \mathrm{Mg} / \mathrm{m}^{3}$

$0.081 \mathrm{~mm}^{-1}$

472

$0.41 \times 0.33 \times 0.12 \mathrm{~mm}^{3}$

3.019 to $30.101^{\circ}$.

$-28<=\mathrm{h}<=28,-6<=\mathrm{k}<=8,-14<=\mid<=14$

19237

$3527[R$ (int) $=0.0595]$

$99.9 \%$

Semi-empirical from equivalents

0.7460 and 0.6594

Full-matrix least-squares on $\mathrm{F}^{2}$

3527 / 0 / 148

1.068

$R 1=0.0507, w R 2=0.1341$

$R 1=0.0711, w R 2=0.1464$

$\mathrm{n} / \mathrm{a}$

0.263 and -0.304 e. $\AA^{-3}$

\section{References}

${ }^{1}$ Sheldrick, G. M. SHELXT. Integrated space-group and crystal-structure determination. Acta Cryst. 2015, A71, 3-8.

${ }^{2}$ Sheldrick, G. M. Crystal structure refinement with SHELXL. Acta Cryst. 2015, C71, 3-8.

${ }^{3}$ Dolomanov O. V.; Bourhis L. J.; Gildea, R. J.; Howard, J. A. K.; Puschmann, H. Olex2: A complete structure solution, refinement and analysis program. J. Appl. Cryst. 2009, 42, 339-341. 